\title{
Supernovae and Gaia
}

\author{
Giuseppe Altavilla ${ }^{1}$ - Maria Teresa Botticella ${ }^{2}$ • \\ Enrico Cappellaro ${ }^{2}$ • Massimo Turatto $^{3}$
}

distinction between classical novae and SNe was made (Baade and Zwicky 1934).

\section{SNe and stellar evolution}

SNe provide natural laboratories for studying the physics of hydrodynamic, nuclear processes in extreme conditions and are involved in the formation of neutron stars, black holes, and gamma-ray bursts (GRBs). Furthermore, SNe are sources of gravitational waves and of neutrino emission, and candidate sites for high-energy cosmic ray acceleration. Thus, it is of broad astrophysical importance to understand the physical conditions for these spectacular explosions.

We recognize two physically defined classes: core collapse-induced explosion of short-lived massive stars (CC SNe) and thermonuclear explosions of long-lived low mass stars (SNe Ia). In recent years deeper and more frequent searches for transient events in the local and distant Universe as well the identification of SN progenitor stars on pre-discovery images from Hubble and Spitzer Space telescopes have provided us with important information on the evolution of massive stars and binary systems (Smart 2009). However, a growing number of peculiar events suggests that the standard scenario of stellar evolution and explosion physics may not be complete and demands further physical understanding and theoretical explanation.

SNe and dark energy

Type Ia SNe are standard candles due to their significant intrinsic brightness and homogeneity. They have provided the first evidence for an accelerated expansion of the Universe and remain one of the more promising probes of the nature and amount of dark energy, (Riess et al. 1998; Perlmutter et al. 1999; Tonry et al. 2003; Astier et al. 2006). CC SNe display a huge range in their physical characteristics, including kinetic energy, radiated energy and the amount of radioactive elements created during the explosion. However, plateau $\mathrm{CC} \mathrm{SNe}$ (SNe IIP) that undergo a long phase of con- 
stant luminosity in their photometric evolution are potential standardizable candles, although they are not as luminous and uniform as SNe Ia (Nugent et al. 2006). Understanding the mechanism that is responsible for the accelerating expansion of the Universe is one of the crucial next steps for cosmology and requires both new searches for type Ia and type IIP SNe to populate the Hubble diagram and the knowledge of their progenitors and explosion mechanisms.

SNe and galaxy evolution

SNe play also a major role in driving the chemical, kinetic, and dynamical evolution of galaxies. They are the main producers of heavy elements and are fundamental for understanding global abundances and abundance patterns in galaxy clusters and in the intergalactic medium (Matteucci and Greggio 1986). SNe Ia are important contributors of iron and release it to the interstellar medium over longer timescales, compared to the $\alpha$ elements produced by CC SNe. Therefore the $\alpha$ to iron abundance ratio can be used to evaluate the formation timescales of stellar systems. Moreover the SN metal-rich ejecta are believed to be a potentially important site of cosmic dust formation. The energy release from $\mathrm{SNe}$ can initiate episodes of star formation (SF), impact the evolution of gas flow and contribute to the feedback processes in galaxies Ceverino and Klypin 2009). A complete and coherent picture of the formation and evolution of the galaxies is a fundamental objective of modern astronomy and SNe are an important ingredient to model the chemical enrichment of the galaxies and the effect of energy/momentum feedback on galaxy formation.

SNe and SFR

The cosmic evolution of $\mathrm{SN}$ rates is an alternative and complementary probe of the SF history (SFH) in the galaxies. Due to the short lifetime of progenitor stars the CC SN rate is directly proportional to the current SFR while type Ia SN rate reflects the convolution of the SFH with the distribution of the delay times between progenitor star formation and explosion (Heger et al. 2003; Greggio 2005). Poor statistics is a major limiting factor for using the CC SN rate as a tracer of the SFR. At low redshift the difficulty is in sampling large enough volumes of the local Universe to ensure significant statistics (e.g. Botticella et al. 2012), while at high redshift the difficulty lies in detecting and typing complete samples of intrinsically faint SNe (Botticella et al. 2008; Bazin et al. 2009; Li et al. 2011c). Moreover it is expected that a fraction of CC SNe are missed by optical searches, since they are embedded in dusty spiral arms or galaxy nuclei. This fraction may change with redshift, if the amount of dust in galaxies evolves with time. Progress in using CC SN rates as SFR tracers requires accurate measurements of rates at various cosmic epochs and in different environments. Furthermore it requires a comparison with other SFR diagnostics in a wide range of redshift to investigate the causes of the observed discrepancy.

Despite the importance of SNe in several astronomical topic many fundamental questions remain with regard these exciting phenomena.

\subsection{SNe}

Stellar evolution theory predicts that all stars more massive than $8-10 M_{\odot}$ complete eso-energetic nuclear burnings and develop an iron core that cannot be supported by any further nuclear fusion reaction, or by electron degenerate pressure. The collapse of the iron core results in the formation of a compact object, a neutron star or a black hole, accompanied by the highvelocity ejection of a large fraction of the progenitor mass. Despite this basic understanding, the picture of massive star death emerging from recent discovery is far to be clear.

Which massive star in which evolutionary stage produce which CC SN type?

Different types of CC SNe have been identified on the basis of their spectroscopic and photometric properties: type II SNe show presence of $\mathrm{H}$ in the spectra, (type IIP SNe exhibit a long plateau in their light curve while type IIL show a linear decline rate), type Ib SNe lack $\mathrm{H}$ but show $\mathrm{He}$, type Ic SNe lack both $\mathrm{H}$ and $\mathrm{He}$, type IIn $\mathrm{SNe}$ exhibit a narrow $H \alpha$ emission line and present other strong evidences of interaction of the ejecta with circumstellar material (CSM) already at early phases of their evolution. This sequence has been explained on the basis of the progenitor mass loss history with the most massive stars loosing the largest fraction of their initial mass (Heger et al. 2003): type IIP progenitor stars are red super giants (RSGs) that explode with most of their $\mathrm{H}$ envelope present, type IIL progenitors had extensive mass loss but preserved a small $\mathrm{H}$ envelope, type $\mathrm{Ib} / \mathrm{c}$ progenitors are Wolf-Rayet stars that had lost their $\mathrm{H}$ and $\mathrm{He}$ envelopes, type IIn progenitor have suffered a major mass loss episode just before the explosion. Several CC SNe with characteristics intermediate between these canonical types have been recently discovered suggesting a smooth transition and requiring the introduction of hybrid classes (for example type IIb SNe and Ibn SNe). The progenitor stars of SNe IIP are the best constrained from direct detection since these $\mathrm{SNe}$ are the most frequent in the nearby Universe. Their mass function seems to suggest a lack of high mass progenitors $\left(>17 M_{\odot}\right)$ and raises 
the intriguing possibility that more massive stars collapse directly to a black-hole and produce either faint or failed SNe (Smartt et al. 2009). The progenitors of $\mathrm{SNe} \mathrm{Ib} / \mathrm{c}$ have so far eluded discovery and several observational evidences have suggested an alternative scenario where a significant fraction of their progenitors are stars of much lower initial mass in close binary systems stripped of their envelopes by the interaction with the companion (Podsiadlowski et al. 2004; Eldridge et al. 2011). Recent observations have suggested the possibility that stars explode as CC SNe shortly after the luminous blue variable (LBV) phase characterized by sporadic variability and occasionally giant outbursts with a huge mass loss in a single short event (Humphreys and Davidson 1994). In particular some SNe (2006jc, 2006gy, Pastorello et al. 2008; Smith et al. 2007; Agnoletto et al. 2009) showed evidence that their progenitors had properties consistent with LBV and in one case a pre-explosion outburst was seen.

Which physical parameters drive the CC SN diversity?

The variety of the observational properties shown by $\mathrm{CC} \mathrm{SNe}$ and the relative numbers of different types reflects the large range of stellar types seen in the upper region of the Hertzsprung Russel Diagram above $8 M_{\odot}$ and the different physical conditions (radius, density profile and surrounding CSM) before the explosion (Hirschi et al. 2010). The simple scheme where only the mass loss drives the evolution of massive stars can not explain the surprising diversity in explosion energies and it is essential to investigate the effect of other key factors (metallicity, rotation rate, magnetic fields, binary membership) (Heger et al. 2005; Eldridge and Tout 2004; Hirschi et al. 2005; Yoon and Langer 2005).

A major puzzle is due to the discovery of a number of SNe at the extrema of the luminosity function and requires further physical understanding. A subset of sub-luminous type II SNe shows both a very faint radioactive tail in the light curve and a low expansion velocity suggesting low energy explosions and a possible black hole remnant, giving rise to significant fallback (Zampieri et al. 2003; Pastorello et al. 2004).

Different types of extremely luminous $\mathrm{CC} \mathrm{SNe}$ have been recently discovered in dwarf, faint host galaxies (Botticella et al. 2010; Quimby et al. 2007; Pastorello et al. 2010) with an enormous luminosity at maximum that can not be powered by radioactive ${ }^{56} \mathrm{Ni}$. Several scenarios have been proposed to explain these events: an explosion driven by black hole accretion (Utrobin et al. 2010), the spin down of a rapidly rotating young magnetar Kasen and Bildsten 2010;
Wooslev 2010), interaction of the SN ejecta with a dense CSM (Blinnikov and Sorokina 2010; Chevalier and Irwin 2011) or pulsational pair instability in which collisions between high velocity shells are the source of multiple, bright optical transients (Wooslev et al. 2007).

What are the alternative explosion mechanisms producing a CC SN?

To explain the full range of explosion parameters of CC SNe some theoretical studies suggested two alternative explosion mechanisms with respect to the collapse of the iron core: electron capture and pair instability. Electron capture $\mathrm{SNe}$ (EC SNe) occur when a star in the mass range of $\sim 8-10 M_{\odot}$ forms an electron degenerate $\mathrm{O}-\mathrm{Ne}-\mathrm{Mg}$ core and the core may collapse before $\mathrm{Ne}$ ignition (Nomoto 1984; Ritossa et al. 1999; Siess 2007; Poelarends et al. 2008). The lower and maximum initial mass for EC SNe is determined by the competing effects of core growth and mass loss during the late evolutionary stages of super-AGB stars and are quite uncertain $\left(\sim 9-9.25 M_{\odot}\right.$, Siess 2007; Poelarends et al. 2008). The pair instability SNe (PI SNe) occur when the high temperatures in a massive core $\left(\right.$ He core $\geq 40 M_{\odot}$ and initial mass $\geq 100 M_{\odot}$ ) induces electron-positron pair production reducing the radiation pressure and hence leading to a rapid contraction followed by a thermonuclear explosion. A similar mechanism is pulsational pair instability in which a massive core undergoes interior instability again due to electron-positron pair production ejecting many solar masses in a series of giant pulses (Woosley et al. 2007). At the moment there are few observational probes of PI SNe.

Which type of massive stars produce black holes?

The origin of stellar mass black holes is not yet understood: some stars $\left(25-40 M_{\odot}\right)$ are expected to produce weak explosions with a black hole formed by fallback, some others $\left(>40 M_{\odot}\right)$ collapse into a black hole directly without any optical signature (failed $\mathrm{SNe}$ ).

The failed SNe have been invoked by theorists because of the difficulties of producing an explosion in analytical models (Heger et al. 2003; Eldridge and Tout 2004, e.g.) but there are remarkably few observational probes of them, e.g. the lack of high mass progenitors may suggest there is a population of blackhole forming SNe which so far have eluded discovery. The detection of sudden neutrino bursts with given duration and energy (to discriminate other events, such as ordinary SNe, neutron stars formations and so on), may be pontentially the signature of failed SNe (Yang and Lunardini 2011; Sumivoshi et al. 2010). Also gamma-ray astronomy (MacFadyen and Woosley 1999) and gravitational-wave astronomy may be sensitive probes of failed SNe (Kotake 2010). 
What is the nature of SN impostors?

The luminosity gap separating novae and sub-luminous SNe is recently being populated by a number of low energy events that have been classified as SNe (SN impostors) and show a significant diversity ranging from giant eruptions of LBVs to very faint transients of unknown physical origin (Smith et al. 2011). The overlapping of bolometric luminosity and kinetic energies between SN impostors and faint $\mathrm{CC}$ SNe requires to investigate in more detail the observational differences between explosive and eruptive transients. At the moment the only certain test to distinguish stellar eruptions from genuine $\mathrm{CC}$ SN explosions is to observe the outcome of the transient event: the surviving progenitor star in the case of eruptive event or a compact SN remnant in the case of CC SN explosion (Chevalier 2005). We also need to understand the diversity of these transients that may reflect different eruption or explosion mechanisms (luminous red novae, stellar mergers in low mass systems, erupting $\mathrm{OH} / \mathrm{IR}$ stars, weak EC SNe from superAGB stars) (Kulkarni et al. 2007; Prieto et al. 2008; Botticella et al. 2009; Kochanek 2011).

What distinguishes a GRB progenitor from that of hypernovae?

The energetically most extreme SNe Ib/Ic (hypernovae) have been associated to long GRBs (Woosley and Bloom 2006; Campana et al. 2008; Modjaz 2011). GRB-SNe are strongly aspherical explosions characterized by a very luminous maximum light, a huge expansion velocity and a very high kinetic energy (about 10 larger than observed in standard CC SNe) (Maeda et al. 2008). Their progenitors are massive stars $\left(30-40 M_{\odot}\right)$ in low metallicity star forming environments (Fruchter et al. 2006; Modiaz et al. 2008) with high rotation rate (Yoon and Langer 2005) that lost all $\mathrm{H}$ envelope. To date only six clear cases of hypernovae have been discovered 11 plus another three cases which show possible SN signatures in the spectra of the GRB afterglows. The existence of SNe Ic/Ib without observed GRBs, as well as that of GRBs without SN signatures, raises the question of what distinguishes a long GRB progenitor from that of a hypernova and an ordinary $\mathrm{SN} \mathrm{Ic/Ib}$ (Della Valle et al. 2006).

${ }^{1} \mathrm{SN} 1998 \mathrm{bw} \&$ GRB $980425(\mathrm{z}=0.0085)$ Galama et al. 1998); SN 2003dh \& GRB $030329 \quad(\mathrm{z}=0.17) \quad$ Stanek et al. 2003; Hiorth et al. 2003; Matheson et al. 2003); SN 2003lw \& GRB $031202(\mathrm{z}=0.1)$ (Malesani et al.|2004); SN 2006aj \& GRB060218 $(z=0.033)$ Campana et al. 2006; Pian et al. 2006); SN 2008hw \& GRB081007 $(\mathrm{z}=0.53)$ (Della Valle et al. 2008), SN2010bh \& GRB100316D at $\mathrm{z}=0.0593$ (Chornock et al. 2010; Starling et al. 2011)

\section{$1.2 \mathrm{SNe}$ Ia}

There is general consensus that SNe Ia are thermonuclear explosions of a carbon and oxygen white dwarf (CO WD) near the Chandrasekhar mass $\left(1.4 M_{\odot}\right)$ in a binary system. The use of SNe Ia as standard candles is based on the assumption that all SNe Ia have similar progenitors and are highly homogeneous but the nature of the companion star and the details of the explosion mechanism are still debated.

What is the progenitor star and explosion mechanism of SNe Ia?

The most widely favoured progenitor scenarios for SNe Ia are the single degenerate scenario (SD) in which a WD, accreting from a non degenerate companion (a main sequence star, a subgiant, a red giant or a helium star), grows in mass until it reaches the Chandrasekhar mass and explodes in a thermonuclear runaway, or double degenerate scenario (DD), in which a close double WD system merges after orbital shrinking due to the emission of gravitational waves (Hillebrandt and Niemever 2000; Maoz and Mannucci 2011).The time elapsed from the birth of the binary system to the SN explosion (delay-time) spans from tens of million years to ten billion years (Greggio 2005). A thermonuclear explosion may occur before the WD reaches the Chandrasekhar mass (sub-Chandrasekhar models) (Fink et al. 2011, and reference therein) or when the WD exceed the Chandrasekhar mass (superChandrasekhar models) in both SD and DD scenario Hachisu et al. 2011). The observed features of standard SNe Ia are better explained by the Chandrasekhar mass although no conclusive evidence for a specific scenario has so far been found. The recent discovery of variable circumstellar absorption lines in the SNIa 2006X (Patat et al. 2007) and the first direct detection of the precursor of a SN Ia (an X-Ray source at the position of SN 2007on, Voss and Nelemans 2008) seem to support the SD scenario. However Roelofs et al. (2008) did not detect any X-ray source in images taken six weeks after SN 2007on optical maximum and found an offset between the SN and the measured X-ray source position of $1.15 \pm 0.27$ ". Only future observations can shed light on the proposed connection between the Xray source and the progenitor of SN 2007on, and thus on the most plausible scenario for this SN Ia progenitor. The analysis of the recent SN 2011fe in M101 seems to rule out the presence of a red giant and helium-star donors in pre-explosion images (Li et al. 2011a), of a circumstellar wind from a giant donor Horesh et al. 2012) and of shocks from ejecta hitting a companion, by analysing very early optical and UV data (Nugent et al. 2011; Brown et al. 2011; Bloom et al. 2012). 
The explosion mechanism of standard $\mathrm{SNe}$ Ia is also largely unknown (see Hillebrandt and Niemeyer 2000 for a review). Key issues include the initiation of the thermonuclear runaway, its initial ignition point and subsequent evolution. In the Chandrasekhar mass scenario, a WD is thought to ignite near the center. At first the flame propagates subsonically as a deflagration and in a second phase a detonation triggers that propagates supersonically. The mechanism that leads to the formation of the detonation is still an open question: detonation transition by turbulence (e.g. Röpke and Niemever 2007) gravitationally confined detonation (e.g. Jordan et al. 2008; Meakin et al. 2009) and pulsating reverse detonation (Bravo and García-Senz 2009).

Why does their brightness correlate with light curve shape, color, stellar population age, metallicity?

Thanks to the uniformity of their peak luminosities SNe Ia can be used as cosmological standard candles. A phenomenological correlation between their absolute magnitude and the shape of their light curves (with brighter objects having a slower rate of decline) has been provided to calibrate their distances (Phillips 1993; Phillips et al. 1999). Only recently models were able to reproduce this calibration in terms of the underlying physics (Kasen et al. 2009). However, this oneparameter description does not completely account for the variety of SNe Ia. The relation between color and light curve shape (brighter SNe Ia are both bluer and have wider light curves than their fainter counterparts) has been exploited to provide a more accurate luminosity calibration. However, the intrinsic color variations of SNe Ia have not yet been fully understood. Several suggestions have been made for additional parameters: metallicity Gallagher et al. 2005; Höflich et al. 2010), high-velocity spectral features, spectral flux ratios (Bailey et al. 2009), and the mass and/or the morphological type of the host galaxy (Kelly et al. 2010; Sullivan et al. 2010). An interesting possibility for the origin of the diverse properties of SNe Ia was recently suggested by Kasen et al. (2009) theoretically and by Maeda et al. (2011) observationally, namely an asymmetry in the SN explosion combined with the observer viewing angle.

What is the origin of SN Ia diversity?

The standard SNe Ia used as cosmological distance indicators make up about $70 \%$ of the observed SNe Ia (Li et al. 2011b). With the advent of the new widearea transient surveys, spectroscopic and photometric peculiarities have been noted in SNe Ia with increasing frequency and new subclasses of SNe Ia Benetti et al. 2005) have been introduced: sub-luminous (e.g. SN 1991bg), normal, bright (e.g. SN 1991T) and superluminous events (e.g. SN 2007if). Whether they form distinct physical groups from normal SNe Ia, with different progenitors and explosion models, or whether they lie at the extreme end of a continuous distribution is unclear. Recent observations of sub-luminous and super-luminous SNe Id 2 suggest that their WD progenitors might have sub-Chandrasekhar and superChandrasekhar mass, respectively (Hicken et al. 2007; Scalzo et al. 2010; Taubenberger et al. 2011). Various scenarios, for example a standard explosion with strong deviations from spherical symmetry of a Chandrasekhar mass WD (Hillebrandt et al. 2007), or alternative energy sources other than radioactivity have been proposed to explain the super-luminous SNe. It is also established that sub-luminous SNe Ia preferably occur in non-star-forming host galaxies with large stellar masses, such as ellipticals (Neill et al. 2009) while super-luminous SNe Ia occur in relatively metal poor host galaxies (Taubenberger et al. 2011) Understanding super-luminous and sub-luminous $\mathrm{SNe}$ Ia has important implications for cosmology and may challenge the paradigm of SN Ia progenitors.

Modern SN searches are revealing ever more extreme examples of faint SNe Ia with lower kinetic energies, the nature of which is controversial with possible models ranging from the direct collapse of a massive star to a black hole to the pure deflagration of a WD (e.g., Folev et al. 2010)

Are there two different progenitor channels? A distinct population of SN Ia progenitors (called prompt) with short delay time (few hundred million years) has been proposed to explain the high SN Ia rate in blue star-forming galaxies and in radio-loud galaxies (Mannucci et al. 2005; Greggio et al. 2008). Tardy SNe Ia arise in old population of at least several Gyr in red, passively evolving ellipticals (Sullivan et al. 2006). The prompt SNe Ia seem to have broader light curves, to be on average brighter and to show fewer intermediatemass elements than their tardy counterparts. The ratio of prompt to tardy events is expected to change with redshift, tracking increasing SFR, with a resulting evolution in average light curve width, SN Ia brightness, and spectral feature strength. The current measurements of type Ia SN rates can not constrain the progenitor models for the uncertainties in the SF history and in SN rate measurements. Progress in investigating the nature of type Ia SN progenitors requires to link SN rates and parent stellar populations with measurements in star forming and in passively evolving galaxies over a wide range of redshifts from $z=0.01$ to $z \sim 4$ Greggio 2010).

${ }^{2}$ SN 2003fg SN 2006gz, SN 2007if, and SN 2009dc 


\section{SN surveys \& Gaia}

\subsection{Historical SN searches}

The first systematic supernova search was undertaken in 1934 by Zwicky using a modest 3-1/4 inch lens camera. In 1936 he continued the search using photographic plates obtained with the 18 inch Schmidt telescope at Palomar Observatory (Baade and Zwicky 1938). This program was followed by the Palomar Supernova Search (Humason and Gates 1960), performed with the 18 and 48 inch Schmidt telescopes, which lasted from 1959 till 1974 and discovered a total of $\simeq 190$ SNe (Zwickv 1938, 1942, 1964).

Several SN searches followed. We list here a few examples: the Asiago SN search, that covered the period 1959-1990, and was performed with the $40 / 50 \mathrm{~cm}$ Schmidt until 1967 and with the 67/92 cm Schmidt afterward. This search produced $\simeq 50 \mathrm{SNe}$, most of them discovered by L. Rosino on photographic plates (Cappellaro et al. 1993, 1997).

The Crimean SN search operated in the period 19611991, using the $40 \mathrm{~cm}$ astrograph of the Sternberg Institute in Crimea, and announced $\simeq 40$ SNe (Tsvetkov 1983).

Reverend Evans visual search produced 24 SNe in the period 1980-1988 using 25-41 cm telescopes van den Bergh 1987; Evans et al. 1989). Evans' SNe were usually bright and nearby objects caught soon after explosion and hence interesting targets for detailed follow-up campaigns.

The Observatoire de la Côte dAzur search was performed analyzing photographic plates acquired for other programmes using the $90 / 152 \mathrm{~cm}$ Schmidt in the period 1987-1994 and produced $\simeq 68$ SNe (Pollas 1994). The Calán Tololo search operated in the period 19891995 using the $60 / 90 \mathrm{~cm}$ Schmidt. The survey discovered $\simeq 54$ SNe (on photographic plates) between 1990 and 1993 (Hamuv et al. 1993). This was the first survey at redshifts suitable for starting cosmological studies $(0.01<z<0.1)$.

\subsection{Modern and future SN searches}

In the last two decades of the $20^{t h}$ century the advent of CCDs revolutionized astronomy and visual or photographic observations were substituted by digital images. Mechanical blinking machines and visual inspections were replaced by computer analysis. The improved CCD and computer technology, the increasing size of telescopes, the advent of larger detectors or CCD mosaics, the development of automated/robotic telescopes and more sophisticated image analysis software boosted

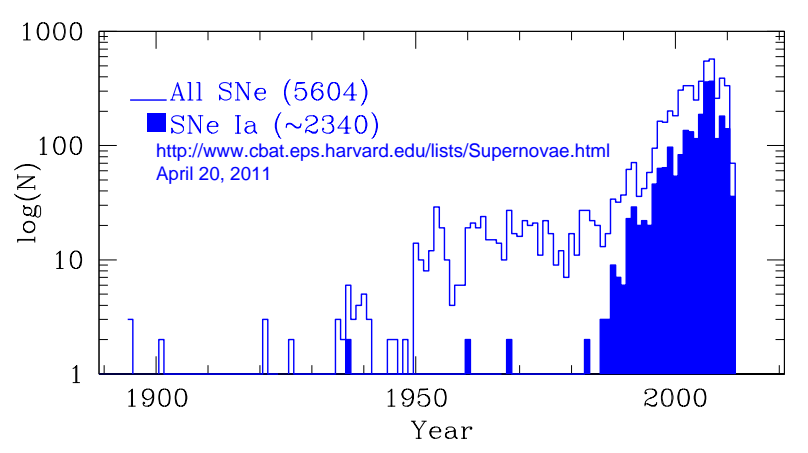

Fig. 1 SN discovery rate with time. The filled histogram refers to type Ia $\mathrm{SNe}$

the productivity of SN surveys of the '90s. Fig. 1 1 graphically shows the evolution of the SN discovery rate with time and the steep increase that started at the end of last century. The same figure shows that a bit less than one half of the total are type Ia SNe. Fig. 2 shows that the number of SNe discovered from 1980 to 2010 increased by a factor $\simeq 10$ with respect to all SN discovered before 19803. We report here a not-exhaustive list of modern supernova surveys to illustrate this boom:

- the High-Z SN search (Schmidt et al. 1998; Riess et al. 1998; Tonry et al. 2003);

et+dile. Supernova Cosmology Project (SCP; Perlmutter et al. 1999; Knop and Supernova Cosmology Project Collaboration 2003);

- the Lick Observatory and the Tenagra Observatory Supernova Searches (LOSS/LOTOSS) (Filippenko et al. 2001; Schwartz et al. 2000; Leaman et al. 2011; Li et al. 2011 b. c); Maoz et al. 2011);

- the Perth Automated SN Search (Williams et al. 1998);

- the Mount Stromlo Abell Cluster Supernova Search (MSACSS; Reiss et al. 1998);

- the Wise Observatory Optical Transients Search (WOOTS; Gal-Yam et al. 1998);

- the CfA Supernova search (Riess et al. 1999);

- the Seoul National University SN Search (SNUSS; Lee et al. 1999);

- the European SN Cosmology Consortium (ESCC; Hardin et al. 1999);

- the QUEST survey (Schaefer et al. 1999; Schaefer 2000);

- the Nearby Galaxies SN Search (NGSS; Strolger et al. 2000);

${ }^{3}$ to be noted that our estimate is based on
the SN announced on CBETS and listed in
http://www.cbat.eps.harvard.edu/lists/Supernovae.html.
not negligible number of SNe discovered by modern searches are
not present in that list.



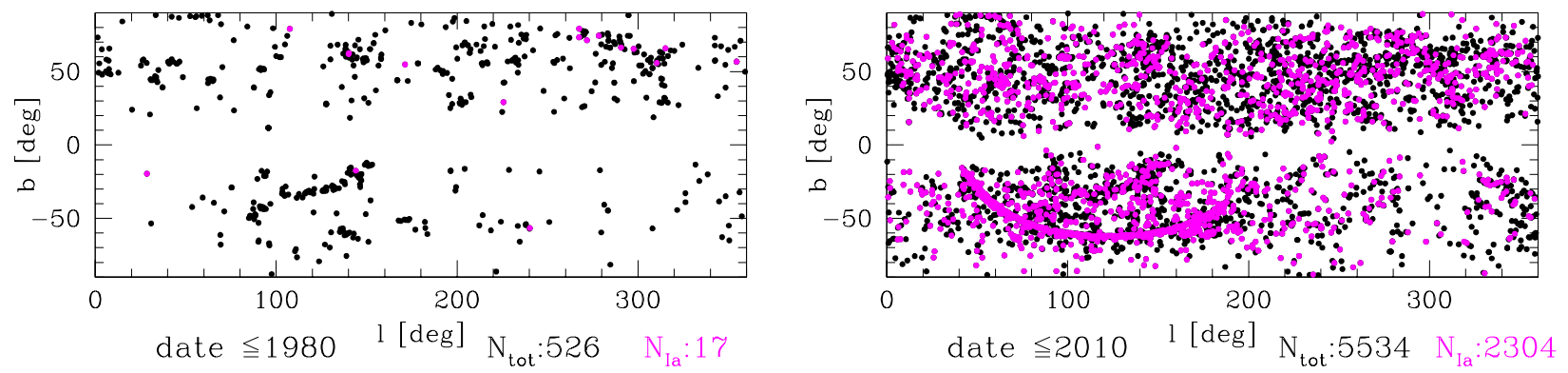

Fig. 2 Sky distribution (galactic coordinates) of the SNe discovered up to 1980 (left panel) and up to 2010 (right panel). Magenta dots: type Ia SNe; black dots: Core Collapse and unknown SNe. Data from http://www.cbat.eps.harvard.edu/lists/Supernovae.html The Milky Way is clearly visible as an empty strip. The arc clearly visible in the right panel is made up by the SDSS supernovae

- the EROS Nearby SN Search (EROSNSS; Hardin et al. 2000);

- the Beijing Astronomical Observatory SN Survey (BAOSS; Qiu et al. 2001);

- the All-sky Southern Supernova Search (Salvo et al. 2003);

- the UK Nova/Supernova Patrol (Hurst 2003);

- the Super Livermore Optical Transient Imaging System (Super-LOTIS; Pérez-Ramírez et al. 2004);

- GOODS HST SN Search (Strolger et al. 2004; Riess et al. 2007);

- The Carnegie Supernova Project (CSP; Hamuv et al. 2006; Phillips 2007; Folatelli et al. 2010);

- the Nearby Supernova Factory (Copin et al. 2006);

- the CFHT Supernova Legacy Survey (SNLS; Astier et al. 2006; Conley et al. 2011);

- SkyMapper (Keller et al. 2007);

- the ESSENCE Supernova Survey (Miknaitis et al. 2007; Wood-Vasey et al. 2007; Foley et al. 2009);

- the Southern inTermediate Redshift ESO Supernova Search (STRESS; Botticella et al. 2008);

- SDSS (Kessler et al. 2009);

- The CHilean Automatic Supernova sEarch (CHASE; Pignata et al. 2009);

- the Palomar Transient Factory (PTF; Law et al. 2009; Rau et al. 2009);

- the DES Supernova Survey (a dedicated Supernova Survey within the Dark Energy Survey, DES (Sako et al. 2011);

- the Catalina Real-Time Transient Survey (CRTS, Diorgovski et al. 2011);

The main characteristic distinguishing the surveys listed above is the targeted redshift range: surveys such as the High-Z SN search, SCP, ESSENCE, CFHT SNLS, GOODS, DES, are focused on high $z$ SNe while other survey look for closer events. Another distinct difference is the observational strategy: galaxy-targeted (usual local) SN surveys, monitoring a selected sample of galaxies or galaxy clusters, such as the LOSS search, and wide field non targeted and unbiased searches such as SDSS. Also some searches are not focused on SN only (as the SDSS, PTF or the CRTS), nevertheless they represent a relevant contribution to the SN discovery rate (see Fig. 2 upper-right panel).

Fig. 3 shows the SN distribution in the sky. As expected from historical reasons, the northern sky is more opulated than the southern, even if the SN distribution is quite homogeneous in galactic coordinates, with about one half of the SN discovered above and below the galactic plane (the Galaxy prevents the detection of SNe behind the disk, that is in fact well visible as an Impty strip in Fig. 21).

In the course of the years the SN searches explored deeper and deeper redshifts. Each redshift range presents different observational difficulties: low redshift surveys $(z<0.1)$ need wide but shallow field observations while pencil beam but deep imaging is enough for high redshift $(z>0.5)$ surveys. Intermediate redshift searches $(0.1 \lesssim z \lesssim 0.5)$ require wide field and deep observations, that have been problematic for a long time. For this reason for some time Hubble diagrams had a gap at the intermediate $z$. Nevertheless filling such interval was important for several reasons: to reduce the statistical uncertainties and to help detecting systematic errors such as a possible evolution of the supernovae characteristics.

The total number of SN announced in the last years is of the order of a few hundred (see Fig. 1) but several surveys are being planned or have just started that are expected to significantly increase the discovery rate of SNe producing (each of them) thousands of detection per years. Some of the most promising next generation surveys are the following: 
- the Panoramic Survey Telescope \& Rapid Response System (PAN-STARRS4), an array of $4 \times 1.8 \mathrm{~m}$ telescopes monitoring $\simeq 30000$ square degrees in the northern hemisphere (Hawaii). This project is expected to detect $10^{7}$ transients in total (SN, GRB etc) and in particular $10^{4}-10^{5} \mathrm{SNe}$ of all types per year (5000 SNe Ia per year at $0<z<1$ ) 5 . The prototype single-mirror telescope PS16 is already operational on Mount Haleakala, Maui, Hawaii Chomiuk et al. 2011).

- the Large Synoptic Survey Telescope (LSST7), a $8.4 \mathrm{~m}$ telescope monitoring $\simeq 20000$ square degrees in the southern hemisphere (Cerro Pachón, Chile). First light is foreseen in 2018, scientific observations will start in 2019 and the survey will be fully operational by 2020. LSST is expected to detect $\simeq 2700$ SNe per night (up to $z \simeq 1.2$ ), 30000 SNe Ia per year at $z<0.38$ and $250000 \mathrm{SNe}$ Ia per year at an average redshift of $\langle z>\simeq 0.45$, but with the most distant $\mathrm{SN}$ up to $z \simeq 0.7$ or even $z \simeq 1.4$ with extended exposure time9. Multiband (six colors) photometry will be provided and a fiber spectrograph can be attached to LSST, but dedicated spectroscopic follow up is required.

These complementary surveys (one in the northern and the other in southern sky) will provide homogeneous unbiased SN samples order of magnitudes larger than the current ones. Multicolour light curves will be secured for each observed object. The bottle-neck of such large surveys will be the rejection of false positive detections, the confirmation of the SN candidates and their follow-up. So far the astronomical community managed to provide substantial spectroscopic follow-up for relatively bright discoveries, but did not provide significant help for slightly fainter (and much more numerous) events (Gal-Yam and Mazzali 2011).

We note the importance of untargeted and unbiased $\mathrm{SN}$ surveys. In the past supernovae searches were usually focused on large galaxies with high star formation rate to maximize the probability of $\mathrm{SN}$ detections. In this case the results were affected by selection effects. The modern unbiased surveys will allow us to detect SNe in faint dwarf galaxies, hostless orphans $\mathrm{SNe}$ and even new SN classes. Many supernovae with peculiar new properties, such as ultra bright SNe, have been

${ }^{4}$ http://pan-starrs.ifa.hawaii.edu/public/home.html
${ }^{5}$ http://www.astro.caltech.edu/ avishay/zwicky1/ppts/-Price
${ }^{6}$ http://ps1sc.org/transients/
${ }^{7}$ http://www.lsst.org/lsst/
${ }^{8}$ http://www.lsst.org/files/docs/aas/2006/WangL.pdf
${ }^{9}$ http://www.lsst.org/lsst/science/scientist_transient
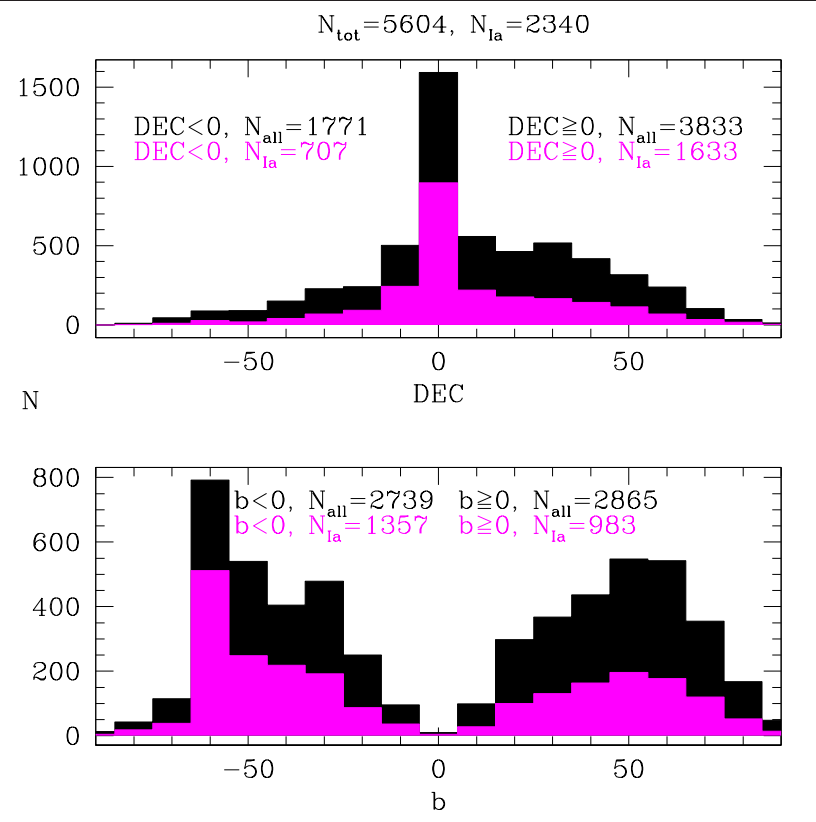

Fig. 3 SN distribution in the sky (upper panel: equatorial coordinates; lower panel galactic coordinates)

already found (e.g. Quimby et al. 2007; Gezari et al. 2009; Pastorello et al. 2010; Chomiuk et al. 2011). We expect that the untargeted surveys to produce an even larger richness in the SN variety that, in turn, will push for new explosion mechanism models.

In this context, a significant contribution will be offered by the ESA space mission Gaia.

\section{Gaia contribution to SN discovery}

Gaid 10 satellite is constituted by two $1.45 \mathrm{~m} \times 0.5 \mathrm{~m}$ telescopes pointing in two directions separated by a $106.5 \mathrm{deg}$ basic angle and merged into a single focal plane. The satellite will operate from L2, $1.5 \times 10^{6} \mathrm{~km}$ from the Earth. Launch date is set in June 2013, the nominal mission ends after 5 years. Gaia will continuously scan the whole sky spinning at $60 " / \mathrm{sec}$. Due to the basic angle, each sky field is observed again after 106.5 minutes 11 and it is possibly re-observed after a full satellite rotation, $4 \mathrm{~h} 13 \mathrm{~m}$ later. This time sampling pattern is repeated sequentially several times as the result of the slow spinning and precession 12 of the satellite. The same field may be observed again after $\sim 30$ days on average and $\sim 80$ times on average over $\sim 30$

\footnotetext{
${ }^{10}$ http://sci.esa.int/science-e/www/area/index.cfm?fareaid=26|

11 and each observation consist on a transit on 9 CCD, with an integration time of $4.4 \mathrm{~s}$ per CCD

12 the precessional period is 63 days long
} 


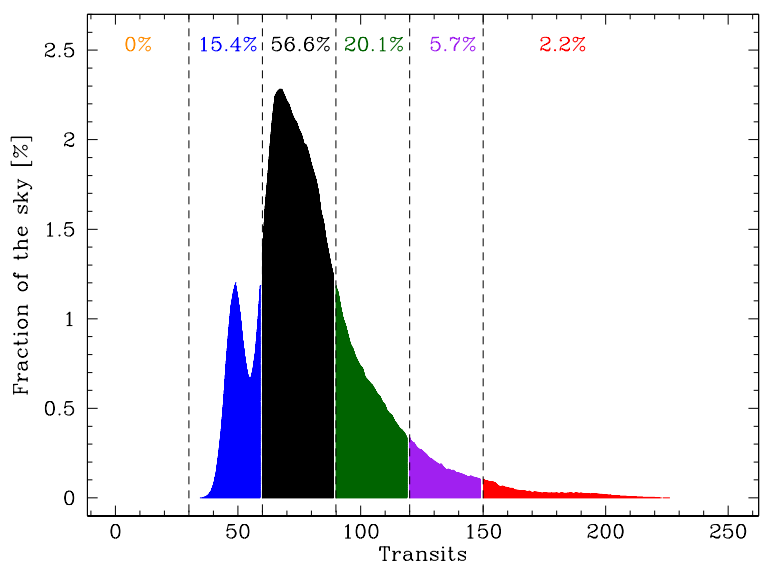

Fig. 4 Fraction of the sky vs the expected end of mission number of transits. About $15.4 \%$ of the sky is expected to be observed $30-60$ times, $\sim 56.6 \%$ of the sky is expected to be observed 60-90 times and $\sim 20.1 \%$ 90-120 times. The remaining $7.9 \%$ is expected to be observed 120-240 times. J. de Bruijne, private communication.

the 5 years, but the scanning law is largely uneven and transits may vary between 40 and 250 times. Regions lying at ecliptic latitude $\pm 45^{\circ}$ will be scanned on average more often than other locations. As shown in Fig. 4 more than one half of the sky will be observed 60-90 times.

Recent estimates of the observable SNe (Type Ia + CC) based on a limiting magnitude $G=19$ predicts $\simeq 6000 \mathrm{SNe}$ over the 5-year mission and the most distant observed type Ia $\mathrm{SNe}$ will be at $\simeq 500 \mathrm{Mpc}$ $(z \simeq 0.12)$. About $1 / 3$ of the $\mathrm{SNe}$ is expected to be observed before maximum.

With the aim to obtain a preliminary characterization of SNe detected by Gaia, we exploit our simulation tool which was developed to estimate the expected number of both SNe types, along with their redshift and apparent magnitude distributions, for any given SN search.

There are two types of inputs for this simulation: the transient properties (photometric evolution and rate of occurrence) and the survey strategy (survey area, limiting magnitude and monitoring cadence).

We considered "classical" SN classes: Ia, IIP, IIL, IIn and Ib/c. For each of these SN types we need to know: a) the template light and color curves along with absolute magnitude at maximum and dispersion ( $\mathrm{Li}$ et al. 2011); b) the K-correction as a function of redshift from the template to the survey observing band (cf. Botticella et al. 2008); c) the current best estimate of the SN rate evolution with redshift (Botticella et al. 2008 and references therein).

$\mathrm{SNe}$ occur in galaxies with a rate that depends on the galaxy type and, for a given type, is, at least in first

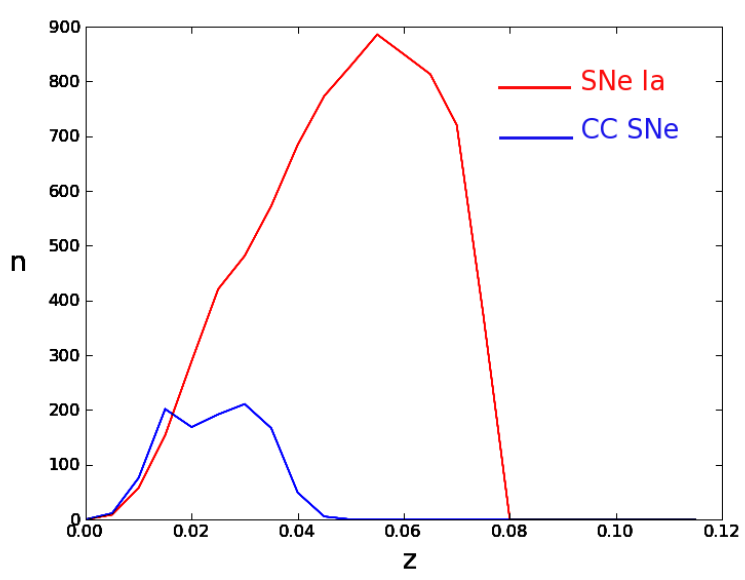

Fig. 5 Redshift distribution of the expected SNe detected by Gaia. Red line: SN Ia, blue line: Core Collapse SNe.

approximation, proportional to the galaxy luminosity. Therefore, in principle, an accurate simulation would require as input the catalog of galaxies monitored by the search along with their type and luminosity. However, if the volume of the search, which is defined by the survey area times its depth, is large enough we can rely on the Universe homogeneity on large scale and adopt volumetric rates. For the current simulation we assume that this is the case for the Gaia survey that, although not very deep, is all sky. We derived the transit distributions for the different sky pixels from the Gaia sky coverage map adopting a pixel resolution of 0.84 square degrees. As a further simplification we assumed that the transits occurring within 6 hours from the first observation (i.e. after a full satellite rotation) see the same sky while more distant transits are evenly distributed within the 5 years of the mission. For our aim to estimate the total number of $\mathrm{SNe}$ detected by Gaia and given the timescale of SN evolution this is quite a good approximation.

We expect that Gaia will detect about $6300 \mathrm{SNe}$, most of them Ia (85\%) and the remaining CC SNe. Of the SNe Ia detected about $30 \%$ will be discovered before maximum light. SNe Ia will be discovered up to redshift $z \simeq 0.1$ with a peak around $z \simeq 0.06$. Instead

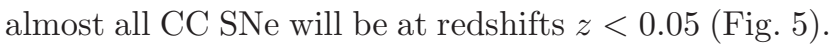
Based on tests with different assumptions, the uncertainty of the simulation is $\sim 10-15 \%$. Our results agree very well with the recent estimates by S. Hodgkin (private communication).

Even if the expected number of SNe detected by Gaia decreased in the most recent estimates (see $\mathrm{Høg}$ et al. 1999; Belokurov and Evans 2003, this work), it still represents a significant contribution to the current discovery rate (Fig 1 ). 


\section{Gaia science alerts and ground based} observations

The Gaia Science Alerts stream 13 , with almost real time transient events, will be the first Gaia data released to the astronomical community. It should be noted that transients will not be proprietary, as is often the case nowadays in many wide-field surveys undertaken by "private" projects. All transients data will be distributed immediately after the detection in accordance with the ESA agreement. This will allow the scientific community to investigate interesting targets immediately. Gaia is expected to disseminate several SN alerts per day along the five years mission, however many of these SN candidates will be either already discovered by other SN survey or very late due to the satellite time sampling. Only the regions with frequently repeated visits will produce SN candidates which are very young, hence potentially most interesting. In all large surveys the rejection of false positive, the confirmation of the SN candidates and their prompt follow-up represent a challenging issue. The Gaia time sampling will provide a photometric confirmation and a partial follow up, since for each observed object will be available a color and a low resolution spectrum provided by the two low dispersion slitless prism spectrographs, the Blue Photometer (BP) and the Red Photometer (RP), that cover the $3300-6800 \AA$ and $6400-10500 \AA$ ranges respectively, with a spectral resolution varying from 20 to 100 approximately. In principle, these data can be very valuable to obtain a prompt transient classification as SN and possibly the SN type. Each object brighter than magnitude 17 will be also observed through the Radial Velocity Spectrometer (RVS), providing an high resolution $(R=11500)$ spectrum in a narrow region (8470$8740 \AA$ ) around the calcium triplet. Unfortunately most of the expected SNe will be fainter than magnitude 17 . Due to the irregular time sampling and to the instrumental setup that is not optimal for SN followup, ground based observations are highly desirable. A prompt follow up is also desirable for studying early phases properly. The astronomical community, including the Italian one, is trying to set up a network to secure a prompt reaction to the announcement of the transients detected by the satellite (Burgon et al. 2011) and an intensive follow-up of the most interesting transients. The most distant SNe Ia accessible to Gaia are at $z \simeq 0.12$, hence $2-4 \mathrm{~m}$ class telescope, or even smaller for brighter objects, located in both the hemispheres, will be suitable. Follow-ups may be limited

${ }^{13}$ see the Gaia Science Alerts Working Group wiki http://www.ast.cam.ac.uk/ioa/research/gsawg to the most intensively regions monitored by Gaia to achieve the maximum efficiency.

Some of us are involved in the Public ESO Spectroscopic Survey for Transient Objects (PESSTO) at NTT and in the Gaia-ESO Survey (GES) at VLT. The goal of PESSTO is to exploit the large allocations of telescope time available through an ESO public survey to assemble data on all forms of SNe covering the full range of parameter space that the current surveys now deliver (luminosity, host metallicity, explosion mechanisms). The survey is expected to deliver time series spectra of 150 transients and optical and NIR light curves for tha majority of these transients. In order to select the targets, $\sim 2000 \mathrm{SNe}$ are expected to be classified, and all information on these will be publicly available, providing an alert list available to the global community. PESSTO will start on April 2012 and 90 nights per year over 4 years (with a possible 1 year extension) have already been granted. The public spectroscopic survey GES started on Dec. 31, 2011 and a total of 240 nights over 4 years (with a possible 1 year extension) have already been awarded to the project. The main goal of the GES is to study the formation and evolution of the Milky Way and its stellar populations. The observation of $\sim 10^{5}$ stars, systematically covering all major components of the Milky-Way will provide valuable complementary data to Gaia and vice versa.

\section{Gaia contribution to SN studies}

The unified study of SN progenitor stars and SN explosion characteristics as well as the analysis of the SN rate evolution with redshift are promising to solve important issues about these intriguing events and to exploit $\mathrm{SNe}$ as cosmological standard candles and as a probe of the SFR. Searches for transients to increase the sample of both standard and peculiar $\mathrm{SNe}$ are required. While transients are not the founding scientific rationale of Gaia, it has become clear that the mission will be a competitive, all sky transient survey without any input object bias and will collect a huge data-set of local SNe, with a significant fraction of under-luminous and overluminous events. A community of interested scientists is being formed and is in contact to discuss the science opportunities with this mission.

The big and homogeneous samples provided by Gaia, coupled with a homogeneous data treatment, will allow robust statistical studies. Relatively rare/peculiar SNe or new transients will be studied in a statistically significant way, subclassifying them into much finer grids if necessary. These large statistical studies will significantly improve our understanding of the SN explosions 
and they will substantially reduce systematic errors in distance estimates, providing tighter constraints on cosmological parameters.

The extremely accurate positions provided by Gaia will allow a detailed study of the SN spatial distribution in different galaxy types and environments. The precise spatial location together with additional data from ground-based observations may provide evidence for possible progenitors and correlations between $\mathrm{SNe}$ properties, location/environment and host type. Distances and redshifts will also be useful to probe the local velocity field. 


\section{References}

Agnoletto, I., Benetti, S., Cappellaro, E., Zampieri, L., Turatto, M., Mazzali, P., Pastorello, A., Della Valle, M., Bufano, F., Harutyunyan, A., Navasardyan, H., EliasRosa, N., Taubenberger, S., Spiro, S., Valenti, S.: Astrophys. J. 691, 1348 (2009). 0810.0635. doi:10.1088/0004$637 X / 691 / 2 / 1348$

Astier, P., Guy, J., Regnault, N., Pain, R., Aubourg, E., Balam, D., Basa, S., Carlberg, R.G., Fabbro, S., Fouchez, D., Hook, I.M., Howell, D.A., Lafoux, H., Neill, J.D., Palanque-Delabrouille, N., Perrett, K., Pritchet, C.J., Rich, J., Sullivan, M., Taillet, R., Aldering, G., Antilogus, P., Arsenijevic, V., Balland, C., Baumont, S., Bronder, J., Courtois, H., Ellis, R.S., Filiol, M., Gonçalves, A.C., Goobar, A., Guide, D., Hardin, D., Lusset, V., Lidman, C., McMahon, R., Mouchet, M., Mourao, A., Perlmutter, S., Ripoche, P., Tao, C., Walton, N.: Astron. Astrophys. 447, 31 (2006). arXiv:astro-ph/0510447. doi:10.1051/0004-6361:20054185

Baade, W., Zwicky, F.: Proceedings of the National Academy of Science 20, 254 (1934). doi:10.1073/pnas.20.5.254

Baade, W., Zwicky, F.: Astrophys. J. 88, 411 (1938). doi:10.1086/143996

Bailey, S., Aldering, G., Antilogus, P., Aragon, C., Baltay, C., Bongard, S., Buton, C., Childress, M., Chotard, N., Copin, Y., Gangler, E., Loken, S., Nugent, P., Pain, R., Pecontal, E., Pereira, R., Perlmutter, S., Rabinowitz, D., Rigaudier, G., Runge, K., Scalzo, R., Smadja, G., Swift, H., Tao, C., Thomas, R.C., Wu, C., The Nearby Supernova Factory: Astron. Astrophys. 500, 17 (2009). 0905.0340. doi:10.1051/0004-6361/200911973

Bazin, G., Palanque-Delabrouille, N., Rich, J., RuhlmannKleider, V., Aubourg, E., Le Guillou, L., Astier, P., Balland, C., Basa, S., Carlberg, R.G., Conley, A., Fouchez, D., Guy, J., Hardin, D., Hook, I.M., Howell, D.A., Pain, R., Perrett, K., Pritchet, C.J., Regnault, N., Sullivan, M., Antilogus, P., Arsenijevic, V., Baumont, S., Fabbro, S., Le Du, J., Lidman, C., Mouchet, M., Mourão, A., Walker, E.S.: Astron. Astrophys. 499, 653 (2009). 0904.1066. doi:10.1051/0004-6361/200911847

Belokurov, V.A., Evans, N.W.: Mon. Not. R. Astron. Soc. 341, 569 (2003). arXiv:astro-ph/0210570. doi:10.1046/j.1365 8711.2003.06427.x

Benetti, S., Cappellaro, E., Mazzali, P.A., Turatto, M., Altavilla, G., Bufano, F., Elias-Rosa, N., Kotak, R., Pignata, G., Salvo, M., Stanishev, V.: Astrophys. J. 623, 1011 (2005). arXiv:astro-ph/0411059. doi:10.1086/428608

Blinnikov, S.I., Sorokina, E.I.: ArXiv e-prints (2010). 1009.4353

Bloom, J.S., Kasen, D., Shen, K.J., Nugent, P.E., Butler, N.R., Graham, M.L., Howell, D.A., Kolb, U., Holmes, S., Haswell, C.A., Burwitz, V., Rodriguez, J., Sullivan, M.: Astrophys. J. Lett. 744, 17 (2012). 1111.0966. doi:10.1088/2041-8205/744/2/L17

Botticella, M.T., Riello, M., Cappellaro, E., Benetti, S., Altavilla, G., Pastorello, A., Turatto, M., Greggio, L., Patat, F., Valenti, S., Zampieri, L., Harutyunyan, A., Pignata, G., Taubenberger, S.: Astron. Astrophys. 479, 49 (2008). 0710.3763. doi:10.1051/0004-6361:20078011
Botticella, M.T., Pastorello, A., Smartt, S.J., Meikle, W.P.S., Benetti, S., Kotak, R., Cappellaro, E., Crockett, R.M., Mattila, S., Sereno, M., Patat, F., Tsvetkov, D., van Loon, J.T., Abraham, D., Agnoletto, I., Arbour, R., Benn, C., di Rico, G., Elias-Rosa, N., Gorshanov, D.L., Harutyunyan, A., Hunter, D., Lorenzi, V., Keenan, F.P., Maguire, K., Mendez, J., Mobberley, M., Navasardyan, H., Ries, C., Stanishev, V., Taubenberger, S., Trundle, C., Turatto, M., Volkov, I.M.: Mon. Not. R. Astron. Soc. 398, 1041 (2009). 0903.1286. doi:10.1111/j.13652966.2009.15082.x

Botticella, M.T., Trundle, C., Pastorello, A., Rodney, S., Rest, A., Gezari, S., Smartt, S.J., Narayan, G., Huber, M.E., Tonry, J.L., Young, D., Smith, K., Bresolin, F., Valenti, S., Kotak, R., Mattila, S., Kankare, E., WoodVasey, W.M., Riess, A., Neill, J.D., Forster, K., Martin, D.C., Stubbs, C.W., Burgett, W.S., Chambers, K.C., Dombeck, T., Flewelling, H., Grav, T., Heasley, J.N., Hodapp, K.W., Kaiser, N., Kudritzki, R., Luppino, G., Lupton, R.H., Magnier, E.A., Monet, D.G., Morgan, J.S., Onaka, P.M., Price, P.A., Rhoads, P.H., Siegmund, W.A., Sweeney, W.E., Wainscoat, R.J., Waters, C., Waterson, M.F., Wynn-Williams, C.G.: Astrophys. J. Lett. 717, 52 (2010). 1001.5427. doi:10.1088/2041-8205/717/1/L52

Botticella, M.T., Smartt, S.J., Kennicutt, R.C., Cappellaro, E., Sereno, M., Lee, J.C.: Astron. Astrophys. 537, 132 (2012). 1111.1692. doi:10.1051/0004-6361/201117343

Bravo, E., García-Senz, D.: Astrophys. J. 695, 1244 (2009). 0901.3008. doi:10.1088/0004-637X/695/2/1244

Brown, P.J., Dawson, K.S., de Pasquale, M., Gronwall, C., Holland, S., Immler, S., Kuin, P., Mazzali, P., Milne, P., Oates, S., Siegel, M.: ArXiv e-prints (2011). 1110.2538

Burgon, R., Wyrzykowski, L., Hodgkin, S.: In: I. N. Evans, A. Accomazzi, D. J. Mink, \& A. H. Rots (ed.) Astronomical Society of the Pacific Conference Series. Astronomical Society of the Pacific Conference Series, vol. 442, p. 243 (2011)

Campana, S., Mangano, V., Blustin, A.J., Brown, P., Burrows, D.N., Chincarini, G., Cummings, J.R., Cusumano, G., Della Valle, M., Malesani, D., Mészáros, P., Nousek, J.A., Page, M., Sakamoto, T., Waxman, E., Zhang, B., Dai, Z.G., Gehrels, N., Immler, S., Marshall, F.E., Mason, K.O., Moretti, A., O'Brien, P.T., Osborne, J.P., Page, K.L., Romano, P., Roming, P.W.A., Tagliaferri, G., Cominsky, L.R., Giommi, P., Godet, O., Kennea, J.A., Krimm, H., Angelini, L., Barthelmy, S.D., Boyd, P.T., Palmer, D.M., Wells, A.A., White, N.E.: Nature 442, 1008 (2006). arXiv:astro-ph/0603279. doi:10.1038/nature04892

Campana, S., Panagia, N., Lazzati, D., Beardmore, A.P., Cusumano, G., Godet, O., Chincarini, G., Covino, S., Della Valle, M., Guidorzi, C., Malesani, D., Moretti, A., Perna, R., Romano, P., Tagliaferri, G.: Astrophys. J. Lett. 683, 9 (2008). 0805.4698. doi:10.1086/591421

Cappellaro, E., Turatto, M., Benetti, S., Tsvetkov, D.Y., Bartunov, O.S., Makarova, I.N.: Astron. Astrophys. 268, 472 (1993)

Cappellaro, E., Turatto, M., Tsvetkov, D.Y., Bartunov, O.S., Pollas, C., Evans, R., Hamuy, M.: Astron. Astrophys. 322, 431 (1997). arXiv:astro-ph/9611191 
Ceverino, D., Klypin, A.: Astrophys. J. 695, 292 (2009). 0712.3285. doi:10.1088/0004-637X/695/1/292

Chevalier, R.A.: Astrophys. J. 619, 839 (2005). arXiv:astroph/0409013. doi:10.1086/426584

Chevalier, R.A., Irwin, C.M.: Astrophys. J. Lett. 729, 6 (2011). 1101.1111. doi:10.1088/2041-8205/729/1/L6

Chomiuk, L., Chornock, R., Soderberg, A.M., Berger, E., Chevalier, R.A., Foley, R.J., Huber, M.E., Narayan, G., Rest, A., Gezari, S., Kirshner, R.P., Riess, A., Rodney, S.A., Smartt, S.J., Stubbs, C.W., Tonry, J.L., WoodVasey, W.M., Burgett, W.S., Chambers, K.C., Czekala, I., Flewelling, H., Forster, K., Kaiser, N., Kudritzki, R.P., Magnier, E.A., Martin, D.C., Morgan, J.S., Neill, J.D., Price, P.A., Roth, K.C., Sanders, N.E., Wainscoat, R.J.: ArXiv e-prints (2011). 1107.3552

Chornock, R., Berger, E., Levesque, E.M., Soderberg, A.M., Foley, R.J., Fox, D.B., Frebel, A., Simon, J.D., Bochanski, J.J., Challis, P.J., Kirshner, R.P., Podsiadlowski, P., Roth, K., Rutledge, R.E., Schmidt, B.P., Sheppard, S.S., Simcoe, R.A.: ArXiv e-prints (2010). 1004.2262

Conley, A., Guy, J., Sullivan, M., Regnault, N., Astier, P., Balland, C., Basa, S., Carlberg, R.G., Fouchez, D., Hardin, D., Hook, I.M., Howell, D.A., Pain, R., Palanque-Delabrouille, N., Perrett, K.M., Pritchet, C.J., Rich, J., Ruhlmann-Kleider, V., Balam, D., Baumont, S., Ellis, R.S., Fabbro, S., Fakhouri, H.K., Fourmanoit, N., González-Gaitán, S., Graham, M.L., Hudson, M.J., Hsiao, E., Kronborg, T., Lidman, C., Mourao, A.M., Neill, J.D., Perlmutter, S., Ripoche, P., Suzuki, N., Walker, E.S.: Astrophys. J. Suppl. Ser. 192, 1 (2011). 1104.1443. doi:10.1088/0067-0049/192/1/1

Copin, Y., Blanc, N., Bongard, S., Gangler, E., Saugé, L., Smadja, G., Antilogus, P., Garavini, G., Gilles, S., Pain, R., Aldering, G., Bailey, S., Lee, B.C., Loken, S., Nugent, P., Perlmutter, S., Scalzo, R., Thomas, R.C., Wang, L., Weaver, B.A., Pécontal, E., Kessler, R., Baltay, C., Rabinowitz, D., Bauer, A.: New Astron. Rev. 50, 436 (2006). doi:10.1016/j.newar.2006.02.035

Della Valle, M., Chincarini, G., Panagia, N., Tagliaferri, G., Malesani, D., Testa, V., Fugazza, D., Campana, S., Covino, S., Mangano, V., Antonelli, L.A., D'Avanzo, P., Hurley, K., Mirabel, I.F., Pellizza, L.J., Piranomonte, S., Stella, L.: Nature 444, 1050 (2006). arXiv:astroph/0608322. doi:10.1038/nature05374

Della Valle, M., Benetti, S., Mazzali, P., Fynbo, J., Pian, E., Filgas, R., Greiner, J., Kruehler, T., Fugazza, D., D'Avanzo, P., Covino, S., Amati, L.: Central Bureau Electronic Telegrams 1602, 1 (2008)

Djorgovski, S.G., Drake, A.J., Mahabal, A.A., Graham, M.J., Donalek, C., Williams, R., Beshore, E.C., Larson, S.M., Prieto, J., Catelan, M., Christensen, E., McNaught, R.H.: ArXiv e-prints (2011). 1102.5004

Eldridge, J.J., Tout, C.A.: Mon. Not. R. Astron. Soc. 353, 87 (2004). arXiv:astro-ph/0405408. doi:10.1111/j.13652966.2004.08041.x

Eldridge, J.J., Langer, N., Tout, C.A.: Mon. Not. R. Astron. Soc. 414, 3501 (2011). 1103.1877. doi:10.1111/j.13652966.2011.18650.x

Evans, R., van den Bergh, S., McClure, R.D.: Astrophys. J. 345, 752 (1989). doi:10.1086/167946
Filippenko, A.V., Li, W.D., Treffers, R.R., Modjaz, M.: In: B. Paczynski, W.-P. Chen, \& C. Lemme (ed.) IAU Colloq. 183: Small Telescope Astronomy on Global Scales. Astronomical Society of the Pacific Conference Series, vol. 246, p. 121 (2001)

Fink, M., Röpke, F.K., Hillebrandt, W., Kromer, M., Sim, S.A., Seitenzahl, I.R.: In: N. V. Pogorelov, E. Audit, \& G. P. Zank (ed.) Astronomical Society of the Pacific Conference Series. Astronomical Society of the Pacific Conference Series, vol. 444, p. 15 (2011)

Folatelli, G., Phillips, M.M., Burns, C.R., Contreras, C., Hamuy, M., Freedman, W.L., Persson, S.E., Stritzinger, M., Suntzeff, N.B., Krisciunas, K., Boldt, L., González, S., Krzeminski, W., Morrell, N., Roth, M., Salgado, F., Madore, B.F., Murphy, D., Wyatt, P., Li, W., Filippenko, A.V., Miller, N.: Astron. J. 139, 120 (2010). 0910.3317. doi:10.1088/0004-6256/139/1/120

Foley, R.J., Matheson, T., Blondin, S., Chornock, R., Silverman, J.M., Challis, P., Clocchiatti, A., Filippenko, A.V., Kirshner, R.P., Leibundgut, B., Sollerman, J., Spyromilio, J., Tonry, J.L., Davis, T.M., Garnavich, P.M., Jha, S.W., Krisciunas, K., Li, W., Pignata, G., Rest, A., Riess, A.G., Schmidt, B.P., Smith, R.C., Stubbs, C.W., Tucker, B.E., Wood-Vasey, W.M.: Astron. J. 137, 3731 (2009). 0811.4424. doi:10.1088/0004-6256/137/4/3731

Foley, R.J., Brown, P.J., Rest, A., Challis, P.J., Kirshner, R.P., Wood-Vasey, W.M.: Astrophys. J. Lett. 708, 61 (2010). 0912.0732. doi:10.1088/2041-8205/708/1/L61

Fruchter, A.S., Levan, A.J., Strolger, L., Vreeswijk, P.M., Thorsett, S.E., Bersier, D., Burud, I., Castro Cerón, J.M., Castro-Tirado, A.J., Conselice, C., Dahlen, T., Ferguson, H.C., Fynbo, J.P.U., Garnavich, P.M., Gibbons, R.A., Gorosabel, J., Gull, T.R., Hjorth, J., Holland, S.T., Kouveliotou, C., Levay, Z., Livio, M., Metzger, M.R., Nugent, P.E., Petro, L., Pian, E., Rhoads, J.E., Riess, A.G., Sahu, K.C., Smette, A., Tanvir, N.R., Wijers, R.A.M.J., Woosley, S.E.: Nature 441, 463 (2006). arXiv:astro-ph/0603537. doi:10.1038/nature04787

Gal-Yam, A., Mazzali, P.: ArXiv e-prints (2011). 1103.5165

Gal-Yam, A., Maoz, D., Dan, J., Ibbetson, P.: IAU Circ. 7055, 1 (1998)

Galama, T.J., Vreeswijk, P.M., van Paradijs, J., Kouveliotou, C., Augusteijn, T., Böhnhardt, H., Brewer, J.P., Doublier, V., Gonzalez, J.-F., Leibundgut, B., Lidman, C., Hainaut, O.R., Patat, F., Heise, J., in't Zand, J., Hurley, K., Groot, P.J., Strom, R.G., Mazzali, P.A., Iwamoto, K., Nomoto, K., Umeda, H., Nakamura, T., Young, T.R., Suzuki, T., Shigeyama, T., Koshut, T., Kippen, M., Robinson, C., de Wildt, P., Wijers, R.A.M.J., Tanvir, N., Greiner, J., Pian, E., Palazzi, E., Frontera, F., Masetti, N., Nicastro, L., Feroci, M., Costa, E., Piro, L., Peterson, B.A., Tinney, C., Boyle, B., Cannon, R., Stathakis, R., Sadler, E., Begam, M.C., Ianna, P.: Nature 395, 670 (1998). arXiv:astro-ph/9806175. doi:10.1038/27150

Gallagher, J.S., Garnavich, P.M., Berlind, P., Challis, P., Jha, S., Kirshner, R.P.: Astrophys. J. 634, 210 (2005). arXiv:astro-ph/0508180. doi:10.1086/491664

Gezari, S., Halpern, J.P., Grupe, D., Yuan, F., Quimby, R., McKay, T., Chamarro, D., Sisson, M.D., Akerlof, C., Wheeler, J.C., Brown, P.J., Cenko, S.B., Rau, A., Djordjevic, J.O., Terndrup, D.M.: Astrophys. J. 690, 1313 (2009). 0808.2812. doi:10.1088/0004-637X/690/2/1313 
Greggio, L.: Astron. Astrophys. 441, 1055 (2005). arXiv:astroph/0504376. doi:10.1051/0004-6361:20052926

Greggio, L.: Mon. Not. R. Astron. Soc. 406, 22 (2010). 1001.3033. doi:10.1111/j.1365-2966.2010.16371.x

Greggio, L., Renzini, A., Daddi, E.: Mon. Not. R. Astron. Soc. 388, 829 (2008). 0805.1512. doi:10.1111/j.13652966.2008.13445.x

Hachisu, I., Kato, M., Saio, H., Nomoto, K.: ArXiv e-prints (2011). 1106.3510

Hamuy, M., Maza, J., Phillips, M.M., Suntzeff, N.B., Wischnjewsky, M., Smith, R.C., Antezana, R., Wells, L.A., Gonzalez, L.E., Gigoux, P., Navarrete, M., Barrientos, F., Lamontagne, R., della Valle, M., Elias, J.E., Phillips, A.C., Odewahn, S.C., Baldwin, J.A., Walker, A.R., Williams, T., Sturch, C.R., Baganoff, F.K., Chaboyer, B.C., Schommer, R.A., Tirado, H., Hernandez, M., Ugarte, P., Guhathakurta, P., Howell, S.B., Szkody, P., Schmidtke, P.C., Roth, J.: Astron. J. 106, 2392 (1993). doi:10.1086/116811

Hamuy, M., Folatelli, G., Morrell, N.I., Phillips, M.M., Suntzeff, N.B., Persson, S.E., Roth, M., Gonzalez, S., Krzeminski, W., Contreras, C., Freedman, W.L., Murphy, D.C., Madore, B.F., Wyatt, P., Maza, J., Filippenko, A.V., Li, W., Pinto, P.A.: Publ. Astron. Soc. Pac. 118, 2 (2006). arXiv:astro-ph/0512039. doi:10.1086/500228

Hardin, D., Walton, N.A., Ellis, R.S., Irwin, M., McMahon, R.G., Hook, I., Ruiz-Lapuente, P., Mendez, J., Visco, A., Astier, P., Balland, C., Blanc, G., Blanchard, A., Fabbro, S., Hammer, F., Letessier-Selvon, A., Levy, J.M., Mouchet, M., Pain, R., Rich, J., Shahmaneche, K., Taillet, R.: IAU Circ. 7182, 1 (1999)

Hardin, D., Afonso, C., Alard, C., Albert, J.N., Amadon, A., Andersen, J., Ansari, R., Aubourg, É., Bareyre, P., Bauer, F., Beaulieu, J.P., Blanc, G., Bouquet, A., Char, S., Charlot, X., Couchot, F., Coutures, C., Derue, F., Ferlet, R., Glicenstein, J.F., Goldman, B., Gould, A., Graff, D., Gros, M., Haissinski, J., Hamilton, J.C., de Kat, J., Kim, A., Lasserre, T., Lesquoy, É., Loup, C., Magneville, C., Mansoux, B., Marquette, J.B., Maurice, É., Milsztajn, A., Moniez, M., Palanque-Delabrouille, N., Perdereau, O., Prévot, L., Regnault, N., Rich, J., Spiro, M., Vidal-Madjar, A., Vigroux, L., Zylberajch, S., The EROS Collaboration: Astron. Astrophys. 362, 419 (2000). arXiv:astro-ph/0006424

Heger, A., Woosley, S.E., Spruit, H.C.: Astrophys. J. 626, 350 (2005). arXiv:astro-ph/0409422. doi:10.1086/429868

Heger, A., Fryer, C.L., Woosley, S.E., Langer, N., Hartmann, D.H.: Astrophys. J. 591, 288 (2003). arXiv:astroph/0212469. doi:10.1086/375341

Hicken, M., Garnavich, P.M., Prieto, J.L., Blondin, S., DePoy, D.L., Kirshner, R.P., Parrent, J.: Astrophys. J. Lett. 669, 17 (2007). 0709.1501. doi:10.1086/523301

Hillebrandt, W., Niemeyer, J.C.: Annu. Rev. Astron. Astrophys. 38, 191 (2000). arXiv:astro-ph/0006305. doi:10.1146/an

Hillebrandt, W., Sim, S.A., Röpke, F.K.: Astron. Astrophys. 465, 17 (2007). arXiv:astro-ph/0702344. doi:10.1051/0004 6361:20077100

Hirschi, R., Meynet, G., Maeder, A.: Astron. Astrophys. 433, 1013 (2005). arXiv:astro-ph/0412454. doi:10.1051/0004 6361:20041554
Hirschi, R., Meynet, G., Maeder, A., Ekström, S., Georgy, C.: In: C. Leitherer, P. Bennett, P. Morris, \& J. van Loon (ed.) Hot and Cool: Bridging Gaps in Massive Star Evolution. Astronomical Society of the Pacific Conference Series, vol. 425, p. 13 (2010)

Hjorth, J., Sollerman, J., Møller, P., Fynbo, J.P.U., Woosley, S.E., Kouveliotou, C., Tanvir, N.R., Greiner, J., Andersen, M.I., Castro-Tirado, A.J., Castro Cerón, J.M., Fruchter, A.S., Gorosabel, J., Jakobsson, P., Kaper, L., Klose, S., Masetti, N., Pedersen, H., Pedersen, K., Pian, E., Palazzi, E., Rhoads, J.E., Rol, E., van den Heuvel, E.P.J., Vreeswijk, P.M., Watson, D., Wijers, R.A.M.J.: Nature 423, 847 (2003). arXiv:astroph/0306347. doi:10.1038/nature01750

Höflich, P., Krisciunas, K., Khokhlov, A.M., Baron, E., Folatelli, G., Hamuy, M., Phillips, M.M., Suntzeff, N., Wang, L., NSF07-SNIa Collaboration: Astrophys. J. 710, 444 (2010). 0912.2231. doi:10.1088/0004-637X/710/1/444

Høg, E., Fabricius, C., Makarov, V.V.: Baltic Astronomy 8, 233 (1999)

Horesh, A., Kulkarni, S.R., Fox, D.B., Carpenter, J., Kasliwal, M.M., Ofek, E.O., Quimby, R., Gal-Yam, A., Cenko, S.B., de Bruyn, A.G., Kamble, A., Wijers, R.A.M.J., van der Horst, A.J., Kouveliotou, C., Podsiadlowski, P., Sullivan, M., Maguire, K., Howell, D.A., Nugent, P.E., Gehrels, N., Law, N.M., Poznanski, D., Shara, M.: Astrophys. J. 746, 21 (2012). 1109.2912. doi:10.1088/0004$637 \mathrm{X} / 746 / 1 / 21$

Humason, M.L., Gates, H.S.: Publ. Astron. Soc. Pac. 72 , 208 (1960). doi:10.1086/127513

Humphreys, R.M., Davidson, K.: Publ. Astron. Soc. Pac. 106, 1025 (1994). doi:10.1086/133478

Hurst, G.M.: Journal of the British Astronomical Association 113, 141 (2003)

Jordan, G.C. IV, Fisher, R.T., Townsley, D.M., Calder, A.C., Graziani, C., Asida, S., Lamb, D.Q., Truran, J.W.: Astrophys. J. 681, 1448 (2008). arXiv:astro-ph/0703573. doi:10.1086/588269

Kasen, D., Bildsten, L.: Astrophys. J. 717, 245 (2010). 0911.0680. doi:10.1088/0004-637X/717/1/245

Kasen, D., Röpke, F.K., Woosley, S.E.: Nature 460, 869 (2009). 0907.0708. doi:10.1038/nature08256

Keller, S., Bessell, M., Schmidt, B., Francis, P.: In: C. Sterken (ed.) The Future of Photometric, Spectrophotometric and Polarimetric Standardization. Astronomical Society of the Pacific Conference Series, vol. 364, p. 177 (2007)

Kelly, P.L., Hicken, M., Burke, D.L., Mandel, K.S., Kirshner, R.P.: Astrophys. J. 715, 743 (2010). 0912.0929. doi:10.1088/0004-637X/715/2/743

Kessler, R., Becker, A.C., Cinabro, D., Vanderplas, J., Frieman, J.A., Marriner, J., Davis, T.M., Dilday, B., Holtzremastrol38Jh191 W., Lampeitl, H., Sako, M., Smith, M., Zheng, C., Nichol, R.C., Bassett, B., Bender, R., Depoy, D.L., Doi, M., Elson, E., Filippenko, A.V., Foley, R.J., Garnavich, P.M., Hopp, U., Ihara, Y., Ketzeback, W., Kollatschny, W., Konishi, K., Marshall, J.L., McMillan, R.J., Miknaitis, G., Morokuma, T., Mörtsell, E., Pan, K., Prieto, J.L., Richmond, M.W., Riess, A.G., Romani, R., Schneider, D.P., Sollerman, J., Takanashi, N., Tokita, K., 
van der Heyden, K., Wheeler, J.C., Yasuda, N., York D.: Astrophys. J. Suppl. Ser. 185, 32 (2009). 0908.4274. doi:10.1088/0067-0049/185/1/32

Knop, R., Supernova Cosmology Project Collaboration: In: American Astronomical Society Meeting Abstracts \#202. Bulletin of the American Astronomical Society, vol. 35, p. 773 (2003)

Kochanek, C.S.: Astrophys. J. 741, 37 (2011). 1106.4722. doi:10.1088/0004-637X/741/1/37

Kotake, K.: In: 25th Texas Symposium on Relativistic Astrophysics, 2010

Kulkarni, S.R., Ofek, E.O., Rau, A., Cenko, S.B., Soderberg, A.M., Fox, D.B., Gal-Yam, A., Capak, P.L., Moon, D.S., Li, W., Filippenko, A.V., Egami, E., Kartaltepe, J., Sanders, D.B.: Nature 447, 458 (2007). 0705.3668. doi:10.1038/nature05822

Law, N.M., Kulkarni, S.R., Dekany, R.G., Ofek, E.O., Quimby, R.M., Nugent, P.E., Surace, J., Grillmair, C.C., Bloom, J.S., Kasliwal, M.M., Bildsten, L., Brown, T., Cenko, S.B., Ciardi, D., Croner, E., Djorgovski, S.G., van Eyken, J., Filippenko, A.V., Fox, D.B., Gal-Yam, A., Hale, D., Hamam, N., Helou, G., Henning, J., Howell, D.A., Jacobsen, J., Laher, R., Mattingly, S., McKenna, D., Pickles, A., Poznanski, D., Rahmer, G., Rau, A., Rosing, W., Shara, M., Smith, R., Starr, D., Sullivan, M. Velur, V., Walters, R., Zolkower, J.: Publ. Astron. Soc. Pac. 121, 1395 (2009). 0906.5350. doi:10.1086/648598

Leaman, J., Li, W., Chornock, R., Filippenko, A.V.: Mon. Not. R. Astron. Soc. 412, 1419 (2011). 1006.4611. doi:10.1111/j.1365-2966.2011.18158.x

Lee, M.G., Park, C., Lee, J., Lee, S., Park, C.G., Jeon, Y.-B., Bolte, M.: IAU Circ. 7237, 3 (1999)

Li, W., Bloom, J.S., Podsiadlowski, P., Miller, A.A., Cenko, S.B., Jha, S.W., Sullivan, M., Howell, D.A., Nugent, P.E., Butler, N.R., Ofek, E.O., Kasliwal, M.M., Richards, J.W., Stockton, A., Shih, H.-Y., Bildsten, L., Shara, M.M., Bibby, J., Filippenko, A.V., Ganeshalingam, M., Silverman, J.M., Kulkarni, S.R., Law, N.M., Poznanski, D., Quimby, R.M., McCully, C., Patel, B., Maguire, K., Shen, K.J.: Nature 480, 348 (2011a). 1109.1593. doi:10.1038/nature10646

Li, W., Leaman, J., Chornock, R., Filippenko, A.V., Poznanski, D., Ganeshalingam, M., Wang, X., Modjaz, M., Jha, S., Foley, R.J., Smith, N.: Mon. Not. R. Astron. Soc. 412, 1441 (2011b). 1006.4612. doi:10.1111/j.13652966.2011.18160.x

Li, W., Chornock, R., Leaman, J., Filippenko, A.V., Poznanski, D., Wang, X., Ganeshalingam, M., Mannucci, F.: Mon. Not. R. Astron. Soc. 412, 1473 (2011c). 1006.4613. doi:10.1111/j.1365-2966.2011.18162.x

MacFadyen, A.I., Woosley, S.E.: Astrophys. J. 524, 262 (1999). arXiv:astro-ph/9810274. doi:10.1086/307790

Maeda, K., Kawabata, K., Mazzali, P.A., Tanaka, M., Valenti, S., Nomoto, K., Hattori, T., Deng, J., Pian, E., Taubenberger, S., Iye, M., Matheson, T., Filippenko, A.V., Aoki, K., Kosugi, G., Ohyama, Y., Sasaki, T., Takata, T.: Science 319, 1220 (2008). 0801.1100. doi:10.1126/science. 1149437

Maeda, K., Leloudas, G., Taubenberger, S., Stritzinger, M., Sollerman, J., Elias-Rosa, N., Benetti, S., Hamuy, M., Folatelli, G., Mazzali, P.A.: Mon. Not. R. Astron. Soc. 413, 3075 (2011). 1101.3935. doi:10.1111/j.13652966.2011.18381.x
Malesani, D., Tagliaferri, G., Chincarini, G., Covino, S., Della Valle, M., Fugazza, D., Mazzali, P.A., Zerbi, F.M., D’Avanzo, P., Kalogerakos, S., Simoncelli, A., Antonelli, L.A., Burderi, L., Campana, S., Cucchiara, A., Fiore, F., Ghirlanda, G., Goldoni, P., Götz, D., Mereghetti, S., Mirabel, I.F., Romano, P., Stella, L., Minezaki, T., Yoshii, Y., Nomoto, K.: Astrophys. J. Lett. 609, 5 (2004). arXiv:astro-ph/0405449. doi:10.1086/422684

Mannucci, F., Della Valle, M., Panagia, N., Cappellaro, E., Cresci, G., Maiolino, R., Petrosian, A., Turatto, M.: Astron. Astrophys. 433, 807 (2005). arXiv:astro-ph/0411450. doi:10.1051/0004-6361:20041411

Maoz, D., Mannucci, F.: ArXiv e-prints (2011). 1111.4492

Maoz, D., Mannucci, F., Li, W., Filippenko, A.V., Della Valle, M., Panagia, N.: Mon. Not. R. Astron. Soc. 412, 1508 (2011). 1002.3056. doi:10.1111/j.1365-2966.2010.16808.x

Matheson, T., Garnavich, P.M., Stanek, K.Z., Bersier, D., Holland, S.T., Krisciunas, K., Caldwell, N., Berlind, P., Bloom, J.S., Bolte, M., Bonanos, A.Z., Brown, M.J.I., Brown, W.R., Calkins, M.L., Challis, P., Chornock, R., Echevarria, L., Eisenstein, D.J., Everett, M.E., Filippenko, A.V., Flint, K., Foley, R.J., Freedman, D.L., Hamuy, M., Harding, P., Hathi, N.P., Hicken, M., Hoopes, C., Impey, C., Jannuzi, B.T., Jansen, R.A., Jha, S., Kaluzny, J., Kannappan, S., Kirshner, R.P., Latham, D.W., Lee, J.C., Leonard, D.C., Li, W., Luhman, K.L., Martini, P., Mathis, H., Maza, J., Megeath, S.T., Miller, L.R., Minniti, D., Olszewski, E.W., Papenkova, M., Phillips, M.M., Pindor, B., Sasselov, D.D., Schild, R., Schweiker, H., Spahr, T., Thomas-Osip, J., Thompson, I., Weisz, D., Windhorst, R., Zaritsky, D.: Astrophys. J. 599, 394 (2003). arXiv:astro-ph/0307435. doi: $10.1086 / 379228$

Matteucci, F., Greggio, L.: Astron. Astrophys. 154, 279 (1986)

Meakin, C.A., Seitenzahl, I., Townsley, D., Jordan, G.C. IV, Truran, J., Lamb, D.: Astrophys. J. 693, 1188 (2009). 0806.4972. doi:10.1088/0004-637X/693/2/1188

Miknaitis, G., Pignata, G., Rest, A., Wood-Vasey, W.M., Blondin, S., Challis, P., Smith, R.C., Stubbs, C.W., Suntzeff, N.B., Foley, R.J., Matheson, T., Tonry, J.L., Aguilera, C., Blackman, J.W., Becker, A.C., Clocchiatti, A., Covarrubias, R., Davis, T.M., Filippenko, A.V., Garg, A., Garnavich, P.M., Hicken, M., Jha, S., Krisciunas, K., Kirshner, R.P., Leibundgut, B., Li, W., Miceli, A., Narayan, G., Prieto, J.L., Riess, A.G., Salvo, M.E., Schmidt, B.P., Sollerman, J., Spyromilio, J., Zenteno, A.: Astrophys. J. 666, 674 (2007). arXiv:astro-ph/0701043. doi:10.1086/519986

Modjaz, M.: Astronomische Nachrichten 332, 434 (2011). 1105.5297. doi:10.1002/asna. 201111562

Modjaz, M., Kewley, L., Kirshner, R.P., Stanek, K.Z., Challis, P., Garnavich, P.M., Greene, J.E., Kelly, P.L., Prieto, J.L.: Astron. J. 135, 1136 (2008). arXiv:astro-ph/0701246. doi:10.1088/0004-6256/135/4/1136

Neill, J.D., Sullivan, M., Howell, D.A., Conley, A., Seibert, M., Martin, D.C., Barlow, T.A., Foster, K., Friedman, P.G., Morrissey, P., Neff, S.G., Schiminovich, D., Wyder, T.K., Bianchi, L., Donas, J., Heckman, T.M., 
Lee, Y.-W., Madore, B.F., Milliard, B., Rich, R.M., Szalay, A.S.: Astrophys. J. 707, 1449 (2009). 0911.0690. doi:10.1088/0004-637X/707/2/1449

Nomoto, K.: Astrophys. J. 277, 791 (1984). doi:10.1086/161749

Nugent, P.E., Sullivan, M., Cenko, S.B., Thomas, R.C., Kasen, D., Howell, D.A., Bersier, D., Bloom, J.S., Kulkarni, S.R., Kandrashoff, M.T., Filippenko, A.V., Silverman, J.M., Marcy, G.W., Howard, A.W., Isaacson, H.T., Maguire, K., Suzuki, N., Tarlton, J.E., Pan, Y.-C., Bildsten, L., Fulton, B.J., Parrent, J.T., Sand, D., Podsiadlowski, P., Bianco, F.B., Dilday, B., Graham, M.L., Lyman, J., James, P., Kasliwal, M.M., Law, N.M., Quimby, R.M., Hook, I.M., Walker, E.S., Mazzali, P., Pian, E., Ofek, E.O., Gal-Yam, A., Poznanski, D.: Nature 480, 344 (2011). 1110.6201. doi:10.1038/nature10644

Nugent, P., Sullivan, M., Ellis, R., Gal-Yam, A., Leonard, D.C., Howell, D.A., Astier, P., Carlberg, R.G., Conley, A., Fabbro, S., Fouchez, D., Neill, J.D., Pain, R., Perrett, K., Pritchet, C.J., Regnault, N.: Astrophys. J. 645, 841 (2006). arXiv:astro-ph/0603535. doi:10.1086/504413

Pastorello, A., Zampieri, L., Turatto, M., Cappellaro, E., Meikle, W.P.S., Benetti, S., Branch, D., Baron, E., Patat, F., Armstrong, M., Altavilla, G., Salvo, M., Riello, M.: Mon. Not. R. Astron. Soc. 347, 74 (2004). arXiv:astroph/0309264. doi:10.1111/j.1365-2966.2004.07173.x

Pastorello, A., Mattila, S., Zampieri, L., Della Valle, M., Smartt, S.J., Valenti, S., Agnoletto, I., Benetti, S., Benn, C.R., Branch, D., Cappellaro, E., Dennefeld, M., Eldridge, J.J., Gal-Yam, A., Harutyunyan, A., Hunter, I., Kjeldsen, H., Lipkin, Y., Mazzali, P.A., Milne, P., Navasardyan, H., Ofek, E.O., Pian, E., Shemmer, O., Spiro, S., Stathakis, R.A., Taubenberger, S., Turatto, M., Yamaoka, H.: Mon. Not. R. Astron. Soc. 389, 113 (2008). 0801.2277. doi:10.1111/j.1365-2966.2008.13602.x

Pastorello, A., Smartt, S.J., Botticella, M.T., Maguire, K., Fraser, M., Smith, K., Kotak, R., Magill, L., Valenti, S., Young, D.R., Gezari, S., Bresolin, F., Kudritzki, R., Howell, D.A., Rest, A., Metcalfe, N., Mattila, S., Kankare, E., Huang, K.Y., Urata, Y., Burgett, W.S., Chambers, K.C., Dombeck, T., Flewelling, H., Grav, T., Heasley, J.N., Hodapp, K.W., Kaiser, N., Luppino, G.A., Lupton, R.H., Magnier, E.A., Monet, D.G., Morgan, J.S., Onaka, P.M., Price, P.A., Rhoads, P.H., Siegmund, W.A., Stubbs, C.W., Sweeney, W.E., Tonry, J.L., Wainscoat, R.J., Waterson, M.F., Waters, C., Wynn-Williams, C.G.: Astrophys. J. Lett. 724, 16 (2010). 1008.2674. doi:10.1088/2041-8205/724/1/L16

Patat, F., Chandra, P., Chevalier, R., Justham, S., Podsiadlowski, P., Wolf, C., Gal-Yam, A., Pasquini, L., Crawford, I.A., Mazzali, P.A., Pauldrach, A.W.A., Nomoto, K., Benetti, S., Cappellaro, E., Elias-Rosa, N., Hillebrandt, W., Leonard, D.C., Pastorello, A., Renzini, A., Sabbadin, F., Simon, J.D., Turatto, M.: Science 317, 924 (2007). 0707.2793. doi:10.1126/science.1143005

Pérez-Ramírez, D., Park, H.S., Williams, G.G., the Superlotis Team: Astronomische Nachrichten 325, 667 (2004). doi:10.1002/asna.200410321

Perlmutter, S., Aldering, G., Goldhaber, G., Knop, R.A., Nugent, P., Castro, P.G., Deustua, S., Fabbro, S., Goobar, A., Groom, D.E., Hook, I.M., Kim, A.G., Kim, M.Y.,
Lee, J.C., Nunes, N.J., Pain, R., Pennypacker, C.R., Quimby, R., Lidman, C., Ellis, R.S., Irwin, M., McMahon, R.G., Ruiz-Lapuente, P., Walton, N., Schaefer, B.,

Boyle, B.J., Filippenko, A.V., Matheson, T., Fruchter, A.S., Panagia, N., Newberg, H.J.M., Couch, W.J., The Supernova Cosmology Project: Astrophys. J. 517, 565 (1999). arXiv:astro-ph/9812133. doi:10.1086/307221

Phillips, M.M.: Astrophys. J. Lett. 413, 105 (1993). doi:10.1086/186970

Phillips, M.M.: In: S. Immler, K. Weiler, \& R. McCray (ed.) Supernova 1987A: 20 Years After: Supernovae and Gamma-Ray Bursters. American Institute of Physics Conference Series, vol. 937, p. 212 (2007). doi:10.1063/1.2803565

Phillips, M.M., Lira, P., Suntzeff, N.B., Schommer, R.A., Hamuy, M., Maza, J.: Astron. J. 118, 1766 (1999). arXiv:astro-ph/9907052. doi:10.1086/301032

Pian, E., Mazzali, P.A., Masetti, N., Ferrero, P., Klose, S., Palazzi, E., Ramirez-Ruiz, E., Woosley, S.E., Kouveliotou, C., Deng, J., Filippenko, A.V., Foley, R.J., Fynbo, J.P.U., Kann, D.A., Li, W., Hjorth, J., Nomoto, K., Patat, F., Sauer, D.N., Sollerman, J., Vreeswijk, P.M., Guenther, E.W., Levan, A., O'Brien, P., Tanvir, N.R., Wijers, R.A.M.J., Dumas, C., Hainaut, O., Wong, D.S., Baade, D., Wang, L., Amati, L., Cappellaro, E., Castro-Tirado, A.J., Ellison, S., Frontera, F., Fruchter, A.S., Greiner, J., Kawabata, K., Ledoux, C., Maeda, K., Møller, P., Nicastro, L., Rol, E., Starling, R.: Nature 442, 1011 (2006). arXiv:astro-ph/0603530. doi:10.1038/nature05082

Pignata, G., Maza, J., Antezana, R., Cartier, R., Folatelli, G., Forster, F., Gonzalez, L., Gonzalez, P., Hamuy, M., Iturra, D., Lopez, P., Silva, S., Conuel, B., Crain, A., Foster, D., Ivarsen, K., Lacluyze, A., Nysewander, M., Reichart, D.: In: G. Giobbi, A. Tornambe, G. Raimondo, M. Limongi, L. A. Antonelli, N. Menci, \& E. Brocato (ed.) American Institute of Physics Conference Series. American Institute of Physics Conference Series, vol. 1111, p. 551 (2009). 0812.4923. doi:10.1063/1.3141608

Podsiadlowski, P., Langer, N., Poelarends, A.J.T., Rappaport, S., Heger, A., Pfahl, E.: Astrophys. J. 612, 1044 (2004). arXiv:astro-ph/0309588. doi:10.1086/421713

Poelarends, A.J.T., Herwig, F., Langer, N., Heger, A.: Astrophys. J. 675, 614 (2008). 0705.4643. doi:10.1086/520872

Pollas, C.: In: S. A. Bludman, R. Mochkovitch, \& J. ZinnJustin (ed.) Supernovae, p. 769 (1994)

Prieto, J.L., Kistler, M.D., Thompson, T.A., Yüksel, H., Kochanek, C.S., Stanek, K.Z., Beacom, J.F., Martini, P., Pasquali, A., Bechtold, J.: Astrophys. J. Lett. 681, 9 (2008). 0803.0324. doi:10.1086/589922

Qiu, Y., Hu, J., Li, W.: In: B. Paczynski, W.-P. Chen, \& C. Lemme (ed.) IAU Colloq. 183: Small Telescope Astronomy on Global Scales. Astronomical Society of the Pacific Conference Series, vol. 246, p. 131 (2001)

Quimby, R.M., Aldering, G., Wheeler, J.C., Höflich, P., Akerlof, C.W., Rykoff, E.S.: Astrophys. J. Lett. 668, 99 (2007). 0709.0302. doi:10.1086/522862

Rau, A., Kulkarni, S.R., Law, N.M., Bloom, J.S., Ciardi, D., Djorgovski, G.S., Fox, D.B., Gal-Yam, A., Grillmair, C.C., Kasliwal, M.M., Nugent, P.E., Ofek, E.O., 
Quimby, R.M., Reach, W.T., Shara, M., Bildsten, L., Cenko, S.B., Drake, A.J., Filippenko, A.V., Helfand, D.J., Helou, G., Howell, D.A., Poznanski, D., Sullivan, M.: Publ. Astron. Soc. Pac. 121, 1334 (2009). 0906.5355. doi:10.1086/605911

Reiss, D.J., Germany, L.M., Schmidt, B.P., Stubbs, C.W.: Astron. J. 115, 26 (1998). arXiv:astro-ph/9710037. doi:10.1086/3

Riess, A.G., Filippenko, A.V., Challis, P., Clocchiatti, A., Diercks, A., Garnavich, P.M., Gilliland, R.L., Hogan, C.J., Jha, S., Kirshner, R.P., Leibundgut, B., Phillips, M.M., Reiss, D., Schmidt, B.P., Schommer, R.A., Smith, R.C., Spyromilio, J., Stubbs, C., Suntzeff, N.B., Tonry, J.: Astron. J. 116, 1009 (1998). arXiv:astro-ph/9805201. doi:10.1086/300499

Riess, A.G., Kirshner, R.P., Schmidt, B.P., Jha, S., Challis, P., Garnavich, P.M., Esin, A.A., Carpenter, C., Grashius, R., Schild, R.E., Berlind, P.L., Huchra, J.P., Prosser, C.F., Falco, E.E., Benson, P.J., Briceño, C., Brown, W.R., Caldwell, N., dell'Antonio, I.P., Filippenko, A.V., Goodman, A.A., Grogin, N.A., Groner, T., Hughes, J.P., Green, P.J., Jansen, R.A., Kleyna, J.T., Luu, J.X., Macri, L.M., McLeod, B.A., McLeod, K.K., McNamara, B.R., McLean, B., Milone, A.A.E., Mohr, J.J., Moraru, D., Peng, C., Peters, J., Prestwich, A.H., Stanek, K.Z., Szentgyorgyi, A., Zhao, P.: Astron. J. 117, 707 (1999). arXiv:astro-ph/9810291. doi:10.1086/300738

Riess, A.G., Strolger, L.-G., Casertano, S., Ferguson, H.C., Mobasher, B., Gold, B., Challis, P.J., Filippenko, A.V., Jha, S., Li, W., Tonry, J., Foley, R., Kirshner, R.P., Dickinson, M., MacDonald, E., Eisenstein, D., Livio, M., Younger, J., Xu, C., Dahlén, T., Stern, D.: Astrophys. J. 659, 98 (2007). arXiv:astro-ph/0611572. doi:10.1086/510378

Ritossa, C., García-Berro, E., Iben, I.J.: Astrophys. J. 515, 381 (1999). doi:10.1086/307017

Roelofs, G., Bassa, C., Voss, R., Nelemans, G.: Mon. Not. R. Astron. Soc. 391, 290 (2008). 0802.2097. doi:10.1111/j.1365 2966.2008.13887.x

Röpke, F.K., Niemeyer, J.C.: Astron. Astrophys. 464, 683 (2007). arXiv:astro-ph/0703378. doi:10.1051/00046361:20066585

Sako, M., Dark Energy Survey (DES) Supernova Working Group, DES Collaboration: In: American Astronomical Society Meeting Abstracts \#217. Bulletin of the American Astronomical Society, vol. 43, p. 205 (2011)

Salvo, M.E., Schmidt, B.P., Ashley, M.C.B., Phillips, A.: Mem. Soc. Astron. Italiana 74, 953 (2003)

Scalzo, R.A., Aldering, G., Antilogus, P., Aragon, C., Bailey, S., Baltay, C., Bongard, S., Buton, C., Childress, M., Chotard, N., Copin, Y., Fakhouri, H.K., Gal-Yam, A., Gangler, E., Hoyer, S., Kasliwal, M., Loken, S., Nugent, P., Pain, R., Pécontal, E., Pereira, R., Perlmutter, S., Rabinowitz, D., Rau, A., Rigaudier, G., Runge, K., Smadja, G., Tao, C., Thomas, R.C., Weaver, B., Wu, C.: Astrophys. J. 713, 1073 (2010). 1003.2217. doi:10.1088/0004$637 X / 713 / 2 / 1073$

Schaefer, B.E.: IAU Circ. 7391, 1 (2000)

Schaefer, B.E., Snyder, J.A., Hernandez, J., Roscherr, B., Deng, M., Ellman, N., Bailyn, C., Rengstorf, A., Smith,
D., Levine, A., Barthelmy, S., Butterworth, P., Hurley, K., Cline, T., Meegan, C., Kouveliotou, C., Kippen, R.M., Park, H.-S., Williams, G.G., Porrata, R., Bionta, R., Hartmann, D., Band, D., Frail, D., Kulkarni, S., Bloom, J., Djorgovski, S., Sadava, D., Chaffee, F., Harris, F., Abad, C., Adams, B., Andrews, P., 308ąa C., Bongiovanni, A., Briceno, C., Bruzual, G., Coppi, P., della Prugna, F., Dubuc, A., Emmet, W., Ferrin, I., Fuenmayor, F., Gebhard, M., Herrera, D., Honeycutt, K., Magris, G., Mateu, J., Mufson, S., Musser, J., Naranjo, O., Oemler, A., Pacheco, R., Paredes, G., Rengel, M., Romero, L., Rosenzweig, P., Sabbey, C., Sánchez, G., Sánchez, G., Schenner, H., Shin, J., Sinnott, J., Sofia, S., Stock, J., Suarez, J., Telléria, D., Vicente, B., Vieira, K., Vivas, K.: Astrophys. J. Lett. 524, 103 (1999). arXiv:astro-ph/9907235. doi:10.1086/312318

Schmidt, B.P., Suntzeff, N.B., Phillips, M.M., Schommer, R.A., Clocchiatti, A., Kirshner, R.P., Garnavich, P., Challis, P., Leibundgut, B., Spyromilio, J., Riess, A.G., Filippenko, A.V., Hamuy, M., Smith, R.C., Hogan, C., Stubbs, C., Diercks, A., Reiss, D., Gilliland, R., Tonry, J., Maza, J., Dressler, A., Walsh, J., Ciardullo, R.: Astrophys. J. 507, 46 (1998). arXiv:astro-ph/9805200. doi:10.1086/306308

Schwartz, M., Li, W.D., Filippenko, A.V., Modjaz, M., Treffers, R.R.: IAU Circ. 7514, 2 (2000)

Siess, L.: Astron. Astrophys. 476, 893 (2007). doi:10.1051/00046361:20078132

Smartt, S.J.: Annu. Rev. Astron. Astrophys. 47, 63 (2009). 0908.0700. doi:10.1146/annurev-astro-082708-101737

Smartt, S.J., Eldridge, J.J., Crockett, R.M., Maund, J.R.: Mon. Not. R. Astron. Soc. 395, 1409 (2009). 0809.0403. doi:10.1111/j.1365-2966.2009.14506.x

Smith, N., Li, W., Foley, R.J., Wheeler, J.C., Pooley, D., Chornock, R., Filippenko, A.V., Silverman, J.M., Quimby, R., Bloom, J.S., Hansen, C.: Astrophys. J. 666, 1116 (2007). arXiv:astro-ph/0612617. doi:10.1086/519949

nith, N., Li, W., Silverman, J.M., Ganeshalingam, M., Filippenko, A.V.: Mon. Not. R. Astron. Soc. 415, 773 (2011). 1010.3718. doi:10.1111/j.1365-2966.2011.18763.x

Stanek, K.Z., Matheson, T., Garnavich, P.M., Martini, P., Berlind, P., Caldwell, N., Challis, P., Brown, W.R., Schild, R., Krisciunas, K., Calkins, M.L., Lee, J.C., Hathi, N., Jansen, R.A., Windhorst, R., Echevarria, L., Eisenstein, D.J., Pindor, B., Olszewski, E.W., Harding, P., Holland, S.T., Bersier, D.: Astrophys. J. Lett. 591, 17 (2003). arXiv:astro-ph/0304173. doi:10.1086/376976

Starling, R.L.C., Wiersema, K., Levan, A.J., Sakamoto, T., Bersier, D., Goldoni, P., Oates, S.R., Rowlinson, A., Campana, S., Sollerman, J., Tanvir, N.R., Malesani, D., Fynbo, J.P.U., Covino, S., D'Avanzo, P., O'Brien, P.T., Page, K.L., Osborne, J.P., Vergani, S.D., Barthelmy, S., Burrows, D.N., Cano, Z., Curran, P.A., de Pasquale, M., D'Elia, V., Evans, P.A., Flores, H., Fruchter, A.S., Garnavich, P., Gehrels, N., Gorosabel, J., Hjorth, J., Holland, S.T., van der Horst, A.J., Hurkett, C.P., Jakobsson, P., Kamble, A.P., Kouveliotou, C., Kuin, N.P.M., Kaper, L., Mazzali, P.A., Nugent, P.E., Pian, E., Stamatikos, M., Thöne, C.C., Woosley, S.E.: Mon. Not. R. Astron. Soc. 411, 2792 (2011). 1004.2919. doi:10.1111/j.13652966.2010.17879.x 
Strolger, L.-G., Smith, R.C., Candia, P., Soderberg, A., Challis, P., Corvarrubias, R., Krick, J., Athey, A., Phillips, M.M., Knopp, R., Conely, A.: IAU Circ. 7404, 1 (2000)

Strolger, L.-G., Riess, A.G., Dahlen, T., Livio, M., Panagia, N., Challis, P., Tonry, J.L., Filippenko, A.V., Chornock, R., Ferguson, H., Koekemoer, A., Mobasher, B., Dickinson, M., Giavalisco, M., Casertano, S., Hook, R., Blondin, S., Leibundgut, B., Nonino, M., Rosati, P., Spinrad, H., Steidel, C.C., Stern, D., Garnavich, P.M., Matheson, T., Grogin, N., Hornschemeier, A., Kretchmer, C., Laidler, V.G., Lee, K., Lucas, R., de Mello, D., Moustakas, L.A., Ravindranath, S., Richardson, M., Taylor, E.: Astrophys. J. 613, 200 (2004). arXiv:astro-ph/0406546. doi:10.1086/422901

Sullivan, M., Le Borgne, D., Pritchet, C.J., Hodsman, A., Neill, J.D., Howell, D.A., Carlberg, R.G., Astier, P., Aubourg, E., Balam, D., Basa, S., Conley, A., Fabbro, S., Fouchez, D., Guy, J., Hook, I., Pain, R., PalanqueDelabrouille, N., Perrett, K., Regnault, N., Rich, J., Taillet, R., Baumont, S., Bronder, J., Ellis, R.S., Filiol, M., Lusset, V., Perlmutter, S., Ripoche, P., Tao, C.: Astrophys. J. 648, 868 (2006). arXiv:astro-ph/0605455. doi:10.1086/506137

Sullivan, M., Conley, A., Howell, D.A., Neill, J.D., Astier, P., Balland, C., Basa, S., Carlberg, R.G., Fouchez, D., Guy, J., Hardin, D., Hook, I.M., Pain, R., PalanqueDelabrouille, N., Perrett, K.M., Pritchet, C.J., Regnault, N., Rich, J., Ruhlmann-Kleider, V., Baumont, S., Hsiao, E., Kronborg, T., Lidman, C., Perlmutter, S., Walker, E.S.: Mon. Not. R. Astron. Soc. 406, 782 (2010). 1003.5119. doi:10.1111/j.1365-2966.2010.16731.x

Sumiyoshi, K., Nakazato, K., Suzuki, H., Yamada, S.: In: Nuclei in the Cosmos., 2010

Taubenberger, S., Benetti, S., Childress, M., Pakmor, R., Hachinger, S., Mazzali, P.A., Stanishev, V., Elias-Rosa, N., Agnoletto, I., Bufano, F., Ergon, M., Harutyunyan, A., Inserra, C., Kankare, E., Kromer, M., Navasardyan, H., Nicolas, J., Pastorello, A., Prosperi, E., Salgado, F., Sollerman, J., Stritzinger, M., Turatto, M., Valenti, S., Hillebrandt, W.: Mon. Not. R. Astron. Soc. 412, 2735 (2011). 1011.5665. doi:10.1111/j.1365-2966.2010.18107.x

Tonry, J.L., Schmidt, B.P., Barris, B., Candia, P., Challis, P., Clocchiatti, A., Coil, A.L., Filippenko, A.V., Garnavich, P., Hogan, C., Holland, S.T., Jha, S., Kirshner, R.P., Krisciunas, K., Leibundgut, B., Li, W., Matheson, T., Phillips, M.M., Riess, A.G., Schommer, R., Smith, R.C., Sollerman, J., Spyromilio, J., Stubbs, C.W., Suntzeff, N.B.: Astrophys. J. 594, 1 (2003). arXiv:astroph/0305008. doi:10.1086/376865

Tsvetkov, D.Y.: Soviet Astron. 27, 22 (1983)

Utrobin, V.P., Chugai, N.N., Botticella, M.T.: Astrophys. J. Lett. 723, 89 (2010). 1009.4425. doi:10.1088/2041$8205 / 723 / 1 /$ L89

van den Bergh, S., McClure, R.D., Evans, R.: Astrophys. J. 323, 44 (1987). doi:10.1086/165806

Voss, R., Nelemans, G.: Nature 451, 802 (2008). 0802.2082. doi: $10.1038 /$ nature06602

Williams, A., Woodings, S., Martin, R., Verveer, A., Biggs, J.: IAU Circ. 6805, 2 (1998)
Wood-Vasey, W.M., Miknaitis, G., Stubbs, C.W., Jha, S., Riess, A.G., Garnavich, P.M., Kirshner, R.P., Aguilera, C., Becker, A.C., Blackman, J.W., Blondin, S., Challis, P., Clocchiatti, A., Conley, A., Covarrubias, R., Davis, T.M., Filippenko, A.V., Foley, R.J., Garg, A., Hicken, M., Krisciunas, K., Leibundgut, B., Li, W., Matheson, T., Miceli, A., Narayan, G., Pignata, G., Prieto, J.L., Rest, A., Salvo, M.E., Schmidt, B.P., Smith, R.C., Sollerman, J., Spyromilio, J., Tonry, J.L., Suntzeff, N.B., Zenteno, A.: Astrophys. J. 666, 694 (2007). arXiv:astroph/0701041. doi:10.1086/518642

Woosley, S.E.: Astrophys. J. Lett. 719, 204 (2010). 0911.0698. doi:10.1088/2041-8205/719/2/L204

Woosley, S.E., Bloom, J.S.: Annu. Rev. Astron. Astrophys. 44, 507 (2006). arXiv:astro-ph/0609142. doi:10.1146/annurev.astro.43.07210

Woosley, S.E., Blinnikov, S., Heger, A.: Nature 450, 390 (2007). 0710.3314. doi:10.1038/nature06333

Yang, L., Lunardini, C.: Phys. Rev. D 84, 063002 (2011). doi:10.1103/PhysRevD.84.063002

Yoon, S.-C., Langer, N.: Astron. Astrophys. 443, 643 (2005). arXiv:astro-ph/0508242. doi:10.1051/0004-6361:20054030

Zampieri, L., Pastorello, A., Turatto, M., Cappellaro, E., Benetti, S., Altavilla, G., Mazzali, P., Hamuy, M.: Mon. Not. R. Astron. Soc. 338, 711 (2003). arXiv:astroph/0210171. doi:10.1046/j.1365-8711.2003.06082.x

Zwicky, F.: Astrophys. J. 88, 529 (1938). doi:10.1086/144007|

Zwicky, F.: Astrophys. J. 96, 28 (1942). doi:10.1086/144430

Zwicky, F.: Annales d'Astrophysique 27, 300 (1964)

This manuscript was prepared with the AAS IATEX macros v5.2. 